\title{
Diagnostic instruments for the assessment of disruptive mood dysregulation disorder: a systematic review of the literature
}

\author{
Ines Mürner-Lavanchy ${ }^{1}$ (D) $\cdot$ Michael Kaess $^{1,2} \cdot$ Julian Koenig $^{1,3}$
}

Received: 25 November 2020 / Accepted: 28 June 2021 / Published online: 7 July 2021

(c) The Author(s) 2021

\begin{abstract}
Disruptive mood dysregulation disorder (DMDD) involves non-episodic irritability and frequent severe temper outbursts in children. Since the inclusion of the diagnosis in the DSM-5, there is no established gold-standard in the assessment of DMDD. In this systematic review of the literature, we provide a synopsis of existing diagnostic instruments for DMDD. Bibliographic databases were searched for any studies assessing DMDD. The systematic search of the literature yielded $K=1167$ hits, of which $n=110$ studies were included. The most frequently used measure was the Kiddie Schedule for Affective Disorders and Schizophrenia DMDD module (25\%). Other studies derived diagnostic criteria from interviews not specifically designed to measure DMDD (47\%), chart review (7\%), clinical diagnosis without any specific instrument (6\%) or did not provide information about the assessment (9\%). Three structured interviews designed to diagnose DMDD were used in six studies $(6 \%)$. Interrater reliability was reported in $36 \%$ of studies (ranging from $\kappa=0.6-1$ ) while other psychometric properties were rarely reported. This systematic review points to a variety of existing diagnostic measures for DMDD with good reliability. Consistent reporting of psychometric properties of recently developed DMDD interviews, as well as their further refinement, may help to ascertain the validity of the diagnosis.
\end{abstract}

Keywords Disruptive mood dysregulation disorder $\cdot$ Irritability $\cdot$ Diagnostics $\cdot$ Measurement $\cdot$ Systematic review of the literature

\section{Introduction}

Disruptive mood dysregulation disorder (DMDD) is a relatively new diagnosis, which has been introduced to the domain of depressive disorders in the fifth version of the Diagnostic and Statistical Manual of Mental Disorders (DSM-5) in 2013 [1]. The diagnosis was endorsed by DSM-5 work groups to address concerns that children with

Ines Mürner-Lavanchy

ines.muerner-lavanchy@upd.unibe.ch; ines@lavanchy.de

$1 \quad$ University Hospital of Child and Adolescent Psychiatry and Psychotherapy, University of Bern, Bolligenstrasse 111, 3000 Bern 60, Switzerland

2 Section for Translational Child and Adolescent Psychiatry, Department of Child and Adolescent Psychiatry, Centre for Psychosocial Medicine, University of Heidelberg, Heidelberg, Germany

3 Section for Experimental Child and Adolescent Psychiatry, Department of Child and Adolescent Psychiatry, Centre for Psychosocial Medicine, University of Heidelberg, Heidelberg, Germany pathological irritability and temper outbursts/anger were being inappropriately diagnosed with bipolar disorder [2]. The diagnosis of bipolar disorder did not accurately capture the non-episodic nature of those children's symptoms and therefore, might have led to questionable treatment decisions [3]. The development of the DMDD diagnosis was based on the description of a broad phenotype of pediatric bipolar disorder called severe mood dysregulation (SMD) by Leibenluft and colleagues in 2003 [4]. In addition to irritability and anger, the latter required symptoms of chronic hyperarousal (e.g. agitation, distractibility, racing thoughts, insomnia, pressured speech or intrusiveness). Increasing evidence of the clinical distinction between episodic and non-episodic irritability and anger as well as distinct pathophysiology finally led to the formulation of the new diagnosis [2, 5-7].

DMDD involves non-episodic anger or irritability and frequent severe temper outbursts over a period of at least one year in pediatric patients aged 6-18 years [1]. Temper outbursts occur on average three or more times per week, can occur verbally or behaviorally (e.g. physical aggression towards objects or persons), their duration or intensity 
is inappropriate to the situation and they are inconsistent with the child's developmental level. DMDD is characterized by persistent irritable and angry mood between temper outbursts in at least two of three settings (i.e. at home, at school, with peers). While the average age of onset is suggested to be 5 years of age [2], the diagnosis is assigned from age 6 , as the identification of pathology before this age is difficult due to normal variations in preschool behavior [8].

The prevalence of DMDD ranges from $0.8 \%$ to $3.3 \%$, with $2-3 \%$ in preschool children, $1-3 \%$ in $9-12$ year-olds, and $0-0.12 \%$ in adolescents [9-11]. Although the prevalence of DMDD decreases with increasing age, individuals with a history of DMDD are at higher risk for adult depression and anxiety, adverse health outcomes, low educational attainment, poverty, and reported police contact, compared to healthy and clinical controls with other psychiatric conditions [11]. Prevalence estimates differ between studies because there is substantial diagnostic variability in the adherence to DSM-5 criteria with respect to the frequency of outbursts, the duration of irritability or the exclusion criteria.

Comorbidity is one of the obstacles which have been reported around the DMDD diagnosis [12]. The majority of patients with DMDD have at least one other comorbid psychiatric disorder, of which oppositional defiant disorder (ODD) or depressive disorders are most commonly reported [10]. In addition, there is substantial diagnostic overlap with childhood psychiatric disorders such as ODD, intermittent explosive disorder or attention deficit hyperactivity disorder (ADHD), questioning the validity of the diagnosis as a distinct disorder [13-15]. Correspondingly, in the International Classification of Diseases and Related Health Problems (ICD-11), DMDD will be listed as a subtype "with chronic irritability-anger" of oppositional defiant disorder [16].

The diagnostic challenges may, at least in part, be due to difficulties in its assessment [17]. As such, symptoms of DMDD are not unique to children referred for psychiatric services. Hence, many existing measures provide questions which assess symptoms relevant to DMDD (e.g. irritability is measured but considered a nonspecific indicator and is related to several other psychiatric disorders) [12]. Moreover, structured interviews or questionnaires specifically developed to diagnose DMDD are still in their infancy. Consequently, there is currently no gold standard or broad consensus regarding the clinical assessment of DMDD.

In this systematic review of the literature, we aimed to provide a synopsis of all measures that have been used in diagnosing DMDD since the advent of the diagnosis in 2013. Study characteristics of the included studies, quantities of used diagnostic measures, and psychometric properties, where applicable, are reported and discussed. The results of this systematic review of the literature might guide future research in the selection of appropriate tools to diagnose DMDD in the clinical and research setting.

\section{Methods}

This systematic review was conducted in accordance with the Preferred Reporting Items for Systematic Review and Meta-Analyses (PRISMA) checklist [18]. The protocol was pre-registered in the International Prospective Register of Systematic Reviews (PROSPERO) and may be accessed under the registration number CRD 42020165496.

\section{Literature search}

The goal of the literature search was to identify any studies assessing DMDD. Therefore, a broad search strategy was formulated. The full electronic search strategy of the systematic literature search in the PubMed database (https:// pubmed.ncbi.nlm.nih.gov) was: ("Disruptive Mood Dysregulation*") OR ("DMDD"). No limits or filters were added to this search. PubMed, Embase, PsycINFO, and Web of Science databases were scrutinized for relevant literature published from 2013 to 31st March 2020. We used identical search terms in all databases. Further, reference lists of publications identified through database search were screened for potentially pertinent studies not identified in the initial search. To reflect the broadest use of tools to diagnose DMDD, in research as well as in the clinic, we included any regular article, case report, or conference abstract published in any of the searched databases.

\section{Study selection}

Studies were excluded if they (a) did not include patients with diagnosed DMDD; or (b) a full text was not available. Prior to a full-text review, the titles, abstracts, and methods sections of the articles identified through database searches were screened for the eligibility criteria outlined above by two independent reviewers until consensus was reached.

\section{Data extraction}

A digital data extraction sheet was developed and refined during the data extraction process. The following data were extracted if available: general information and identifying features of the study, i.e., full reference, year of publication, and country of study origin. Additionally, the article type was identified, comprising regular articles, conference abstracts, or case reports. All article types were included to cover the full breadth of tools available for research and clinical purposes. Magnitudes and percentages of all outcome variables were given for all study types included as 
well as for abstracts only. Further data extracted comprised details on the study design, study population, sample size, and age range. The main outcome was the tool used to diagnose DMDD, including the rater (clinician, parent, self) and whether psychometric properties had been assessed. Where possible, information about the number of items, administration time, and availability of the tool (licensed vs. free of cost) in different languages was obtained. Authors were contacted to provide details if any of the information of interest was not provided in the study.

\section{Results}

\section{Search results}

The first literature search, conducted on January 22, 2020, yielded $K=1149$ records (PubMed $k=168$, PsycINFO $k=471$, Web of Science $k=201$, Embase $k=309$ ). Search updates identical to the first search were carried out on May 26, 2020, yielding an additional $k=18$ records. $K=351$ duplicates were removed from the $K=1167$ records screened for eligibility. Of the $k=172$ full-text articles screened for eligibility, a further $k=53$ studies were excluded as they did not include patients with diagnosed DMDD and $k=9$ because a full text was not obtainable. The PRISMA flow diagram of the full process of study selection is depicted in Fig. 1.

\section{Included studies}

From the initial base of records, $k=110$ studies fulfilled all inclusion criteria and were retained for qualitative syntheses.

General study characteristics of the included studies are described in Table 1. Of the included studies, $k=58$ were regular articles $(52.7 \%)$, while there were $k=41$ conference abstracts $(37.3 \%)$ and $k=11$ case reports $(10.0 \%)$. Most of the studies included a clinical sample $(k=83,75.5 \% ; k=36$ abstracts, $32.7 \%)$, some were population-based $(k=12$, $10.9 \% ; k=2$ abstracts, $1.8 \%)$, case studies $(k=11,10.0 \%$; $k=0$ abstracts), cohort studies ( $k=3,2.7 \%$; $k=1$ abstract, $0.9 \%)$ and $k=1$ study was among youth in the juvenile justice setting ( $0.9 \% ; k=0$ abstracts). $K=85$ studies included unique samples (77.3\%; $k=30$ abstracts), while $k=25$ articles $(22.7 \% ; k=11$ abstracts) reported data from overlapping samples (see Table 1 for details). $K=86$ studies were
Fig. 1 PRISMA flow diagram depicting the study selection process

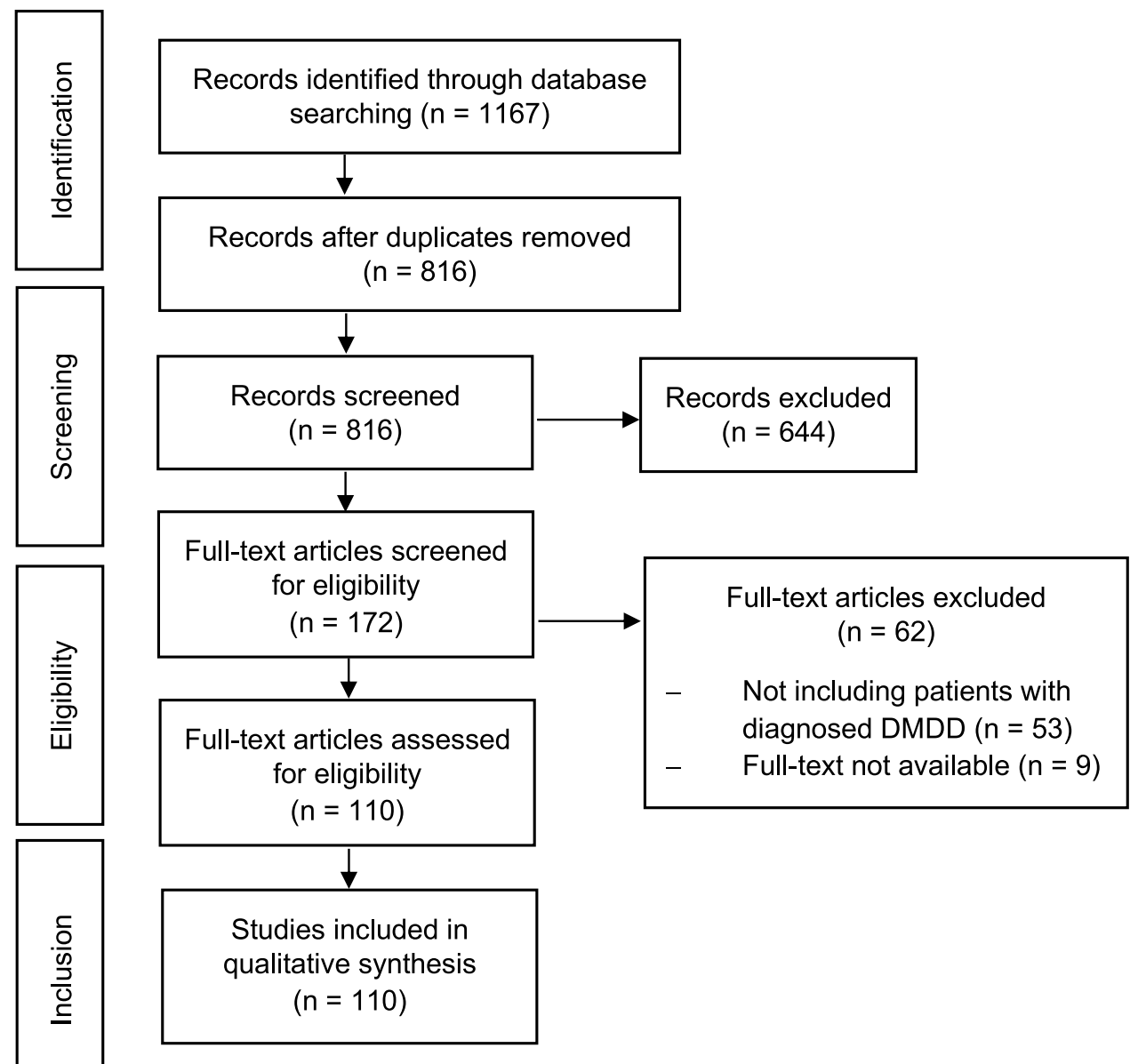


Table 1 Study characteristics by year of publication

\begin{tabular}{|c|c|c|c|c|c|c|c|c|}
\hline Authors & Year & $\begin{array}{l}\text { Country of } \\
\text { origin }\end{array}$ & Article type & Study type & Study design & Sample & $N(\%$ female $)$ & Age (range) $)^{5}$ \\
\hline $\begin{array}{l}\text { Copeland et al. } \\
\text { [10] }\end{array}$ & 2013 & USA & Regular article & $\begin{array}{l}\text { Population- } \\
\text { based }\end{array}$ & Prospective & Community & $3258(50)$ & $2-17$ \\
\hline $\begin{array}{l}\text { Copeland et al. } \\
\text { [11] }\end{array}$ & 2014 & USA & Regular article & $\begin{array}{l}\text { Population- } \\
\text { based }\end{array}$ & Prospective $^{2}$ & Population & $1420(47)$ & $10-25$ \\
\hline $\begin{array}{l}\text { Dougherty et al. } \\
\text { [54] }\end{array}$ & 2014 & USA & Regular article & $\begin{array}{l}\text { Population- } \\
\text { based }\end{array}$ & Prospective & Community & $462(46)$ & 6 \\
\hline $\begin{array}{l}\text { Parmar et al. } \\
\text { [55] }\end{array}$ & 2014 & USA & Case report & Case study & Retrospective & Outpatients & $1(0)$ & 15 \\
\hline Roy et al. [43] & 2014 & USA & Case report & Case study & Retrospective & Outpatients & $1(0)$ & 8 \\
\hline $\begin{array}{l}\text { Sparks et al. } \\
{[56]}\end{array}$ & 2014 & USA & Regular article & Clinical & Prospective $^{2}$ & $\begin{array}{l}\text { Outpatients and } \\
\text { community } \\
\text { controls }\end{array}$ & 616 (NA) & $6-17$ \\
\hline $\begin{array}{l}\text { Deveney et al. } \\
\text { [57] }\end{array}$ & 2015 & USA & Regular article & Clinical & Prospective $^{2}$ & Outpatients & $194(35)$ & $7-17$ \\
\hline $\begin{array}{c}\text { Estrada Prat } \\
\text { et al. [58] }\end{array}$ & 2015 & Spain & $\begin{array}{l}\text { Conference } \\
\text { abstract }\end{array}$ & Clinical & Prospective & Outpatients & $8(25)$ & $7-18$ \\
\hline $\begin{array}{l}\text { Mitchell et al. } \\
\text { [59] }\end{array}$ & 2015 & Canada & $\begin{array}{c}\text { Conference } \\
\text { abstract }\end{array}$ & Clinical & Prospective & Outpatients & $116(\mathrm{NA})$ & NA \\
\hline $\begin{array}{l}\text { Schilpzand } \\
\text { et al. }^{\text {b }}[60]\end{array}$ & 2015 & Australia & $\begin{array}{c}\text { Conference } \\
\text { abstract }\end{array}$ & Clinical & Prospective & patients & $179(31)$ & $6-8$ \\
\hline $\begin{array}{l}\text { Stoddard et al. } \\
\text { [61] }\end{array}$ & 2015 & USA & $\begin{array}{c}\text { Conference } \\
\text { abstract }\end{array}$ & Clinical & Prospective & $\begin{array}{l}\text { patients and } \\
\text { healthy con- } \\
\text { trols }\end{array}$ & $89(48)^{4}$ & $8-18$ \\
\hline Tseng et al. [62] & 2015 & USA & $\begin{array}{l}\text { Conference } \\
\text { abstract }\end{array}$ & Clinical & Prospective & $\begin{array}{l}\text { patients and } \\
\text { healthy con- } \\
\text { trols }\end{array}$ & $75(53)$ & $8-18$ \\
\hline Uran et al. ${ }^{\mathrm{c}}[63]$ & 2015 & Turkey & Regular article & Clinical & Prospective & $\begin{array}{l}\text { Outpatients } \\
\text { and healthy } \\
\text { controls }\end{array}$ & $99(51)$ & $7-18$ \\
\hline Uran et al. ${ }^{\mathrm{c}}[64]$ & 2015 & Turkey & $\begin{array}{c}\text { Conference } \\
\text { abstract }\end{array}$ & Clinical & Prospective & $\begin{array}{l}\text { patients and } \\
\text { healthy con- } \\
\text { trols }\end{array}$ & $99(51)$ & $7-18$ \\
\hline Althoff et al. [9] & 2016 & USA & Regular article & $\begin{array}{l}\text { Population- } \\
\text { based }\end{array}$ & Prospective & Population & $6483(51)$ & $13-18$ \\
\hline $\begin{array}{l}\text { Averna et al. } \\
\text { [65] }\end{array}$ & 2016 & Italy & Case report & Case study & Retrospective & Outpatient & $1(0)$ & 11 \\
\hline $\begin{array}{l}\text { Baweja et al. } \\
\text { [66] }\end{array}$ & 2016 & USA & Regular article & Clinical & Prospective $^{2}$ & Outpatients & $38(28)$ & $7-12$ \\
\hline $\begin{array}{l}\text { Brotman et al. } \\
\text { [67] }\end{array}$ & 2016 & USA & $\begin{array}{c}\text { Conference } \\
\text { abstract }\end{array}$ & Clinical & Prospective & $\begin{array}{l}\text { Patients and } \\
\text { healthy con- } \\
\text { trols }\end{array}$ & $110(45)$ & $9-19$ \\
\hline $\begin{array}{l}\text { Carlson et al. } \\
\text { [68] }\end{array}$ & 2016 & USA & $\begin{array}{c}\text { Conference } \\
\text { abstract }\end{array}$ & Clinical & Prospective & Community & $36(56)$ & 6,9 and 12 \\
\hline $\begin{array}{l}\text { Copeland et al. } \\
\text { [37] }\end{array}$ & 2016 & USA & $\begin{array}{c}\text { Conference } \\
\text { abstract }\end{array}$ & Clinical & Prospective $^{2}$ & Community & 112 (NA) & M 11.4 \\
\hline $\begin{array}{l}\text { Dougherty } \\
\text { et al. }^{\mathrm{d}}[69]\end{array}$ & 2016 & USA & Regular article & $\begin{array}{l}\text { Population- } \\
\text { based }\end{array}$ & Prospective & Population & $473(46)$ & 36 and 9 \\
\hline $\begin{array}{l}\text { Freeman et al. } \\
\text { [70] }\end{array}$ & 2016 & USA & Regular article & Clinical & Prospective $^{2}$ & Outpatients & $597(39)$ & $6-18$ \\
\hline $\begin{array}{l}\text { Fristad et al. } \\
\text { [71] }\end{array}$ & 2016 & USA & Regular article & Clinical & Prospective & Patients & $217(38)$ & $6-12$ \\
\hline Gold et al. [72] & 2016 & USA & Regular article & Clinical & Prospective & $\begin{array}{l}\text { Community, } \\
\text { outpatients } \\
\text { and healthy } \\
\text { controls }\end{array}$ & $184(40)$ & $8-19$ \\
\hline
\end{tabular}


Table 1 (continued)

\begin{tabular}{|c|c|c|c|c|c|c|c|c|}
\hline Authors & Year & $\begin{array}{l}\text { Country of } \\
\text { origin }\end{array}$ & Article type & Study type & Study design & Sample & $N(\%$ female $)$ & Age (range) $)^{5}$ \\
\hline $\begin{array}{l}\text { Kessel et al. } \\
\text { [39] }\end{array}$ & 2016 & USA & Regular article & $\begin{array}{l}\text { Population- } \\
\text { based }\end{array}$ & Prospective & Community & $373(45)$ & 9 \\
\hline Kilic et al. [73] & 2016 & Turkey & Case report & Case study & Retrospective & Outpatient & $1(0)$ & 18 \\
\hline $\begin{array}{l}\text { Mitchell et al. }{ }^{\mathrm{a}} \\
{[74]}\end{array}$ & 2016 & Canada & Regular article & Clinical & Prospective & Outpatients & $108(68)$ & $13-19$ \\
\hline $\begin{array}{l}\text { Mulraney et al. } \\
\text { [75] }\end{array}$ & 2016 & Australia & Regular article & Clinical & Prospective & Community & $179(25)$ & $6-8$ \\
\hline Pogge et al. [76] & 2016 & USA & $\begin{array}{l}\text { Conference } \\
\text { abstract }\end{array}$ & Clinical & Prospective & Inpatients & 100 (NA) & $6-12$ \\
\hline $\begin{array}{l}\text { Stoddard et al. } \\
\text { [77] }\end{array}$ & 2016 & USA & Regular article & Clinical & Prospective & $\begin{array}{l}\text { Patients and } \\
\text { healthy con- } \\
\text { trols }\end{array}$ & $89(48)^{3}$ & $8-18$ \\
\hline Stoddard $^{\mathrm{e}}[78]$ & 2016 & USA & $\begin{array}{c}\text { Conference } \\
\text { abstract }\end{array}$ & Clinical & Prospective & $\begin{array}{l}\text { Patients and } \\
\text { healthy Con- } \\
\text { trols }\end{array}$ & $115(44)$ & $8-17$ \\
\hline $\begin{array}{l}\text { Taskiran et al. } \\
\text { [79] }\end{array}$ & 2016 & Turkey & $\begin{array}{l}\text { Conference } \\
\text { abstract }\end{array}$ & Clinical & Prospective & Outpatients & 29 (NA) & M 9.2 \\
\hline $\begin{array}{l}\text { Tiwari et al. } \\
\text { [80] }\end{array}$ & 2016 & India & Regular article & Clinical & Prospective & Inpatients & $70(24)$ & $6-16$ \\
\hline Topal et al. ${ }^{\mathrm{f}}[81]$ & 2016 & Turkey & $\begin{array}{c}\text { Conference } \\
\text { abstract }\end{array}$ & Clinical & Prospective & Outpatients & $90(48)$ & $12-16$ \\
\hline Topal et al. ${ }^{\mathrm{f}}$ [82] & 2016 & Turkey & $\begin{array}{l}\text { Conference } \\
\text { abstract }\end{array}$ & Clinical & Prospective & $\begin{array}{l}\text { Offspring of } \\
\text { parents with } \\
\text { mood disorder }\end{array}$ & $87(43)$ & $12-16$ \\
\hline Tudor et al. [83] & 2016 & USA & Case report & Case study & Retrospective & Patients & $1(100)$ & 9 \\
\hline Tufan et al. [84] & 2016 & Turkey & Regular article & Clinical & Retrospective & Outpatients & 403 (NA) & $6-17$ \\
\hline $\begin{array}{l}\text { Wiggins et al. } \\
\text { [41] }\end{array}$ & 2016 & USA & Regular article & Clinical & Prospective & $\begin{array}{l}\text { Outpatients } \\
\text { and healthy } \\
\text { controls }\end{array}$ & $71(40)$ & $9-21$ \\
\hline $\begin{array}{l}\text { Alexander et al. } \\
\text { [85] }\end{array}$ & 2017 & USA & $\begin{array}{c}\text { Conference } \\
\text { abstract }\end{array}$ & $\begin{array}{l}\text { Population- } \\
\text { based }\end{array}$ & Prospective & Population & 500 (NA) & $5-21$ \\
\hline $\begin{array}{l}\text { Dougherty } \\
\text { et al. }^{\mathrm{d}}[86]\end{array}$ & 2017 & USA & Regular article & Clinical & Prospective & Community & $329(51)$ & 6 and 9 \\
\hline $\begin{array}{c}\text { Estrada Prat } \\
\text { et al. [87] }\end{array}$ & 2017 & Spain & Regular article & Clinical & Prospective & Patients & $35(33)$ & $6-18$ \\
\hline Eyre et al..$^{\mathrm{g}}[88]$ & 2017 & UK & Regular article & Clinical & Prospective & Patients & $696(16)$ & $6-18$ \\
\hline $\begin{array}{l}\text { Faheem et al. } \\
\text { [89] }\end{array}$ & 2017 & USA & Regular article & Clinical & Retrospective & Inpatients & 490 (NA) & $6-18$ \\
\hline $\begin{array}{l}\text { Higdon et al. } \\
\text { [90] }\end{array}$ & 2017 & USA & $\begin{array}{l}\text { Conference } \\
\text { abstract }\end{array}$ & Clinical & Prospective & $\begin{array}{l}\text { Overweight } \\
\text { patients }\end{array}$ & $438(52)$ & $7-19$ \\
\hline Jain [91] & 2017 & India & $\begin{array}{c}\text { Conference } \\
\text { abstract }\end{array}$ & Clinical & Prospective & Patients & $25(12)$ & $6-9$ \\
\hline $\begin{array}{r}\text { Jalnapurkar } \\
\text { et al. [92] }\end{array}$ & 2017 & USA & $\begin{array}{c}\text { Conference } \\
\text { abstract }\end{array}$ & Clinical & Prospective & Inpatients & 95 (NA) & $8-17$ \\
\hline $\begin{array}{l}\text { Kircanski et al. }^{\mathrm{h}} \\
\text { [93] }\end{array}$ & 2017 & USA & $\begin{array}{c}\text { Conference } \\
\text { abstract }\end{array}$ & Clinical & Prospective & Outpatients & $197(46)$ & $8-18$ \\
\hline $\begin{array}{l}\text { Kircanski et al. } \\
\text { [94] }\end{array}$ & 2017 & USA & $\begin{array}{c}\text { Conference } \\
\text { abstract }\end{array}$ & Clinical & Prospective & $\begin{array}{l}\text { Outpatients } \\
\text { and healthy } \\
\text { controls }\end{array}$ & $199(54)$ & $8-18$ \\
\hline Le et al. [95] & 2017 & USA & $\begin{array}{c}\text { Conference } \\
\text { abstract }\end{array}$ & Clinical & Retrospective & Patients & 7268 (NA) & $<18$ \\
\hline $\begin{array}{l}\text { Martin et al. } \\
\text { [96] }\end{array}$ & 2017 & USA & Regular article & Clinical & Prospective & Outpatients & $139(25)$ & $4-5$ \\
\hline
\end{tabular}


Table 1 (continued)

\begin{tabular}{|c|c|c|c|c|c|c|c|c|}
\hline Authors & Year & $\begin{array}{l}\text { Country of } \\
\text { origin }\end{array}$ & Article type & Study type & Study design & Sample & $N(\%$ female $)$ & Age (range) $)^{5}$ \\
\hline $\begin{array}{l}\text { Matthews et al. } \\
\text { [97] }\end{array}$ & 2017 & USA & $\begin{array}{c}\text { Conference } \\
\text { abstract }\end{array}$ & Clinical & Prospective & $\begin{array}{l}\text { Previous inpa- } \\
\text { tients }\end{array}$ & $91(43)$ & $6-17$ \\
\hline $\begin{array}{l}\text { McTate et al. } \\
\text { [53] }\end{array}$ & 2017 & USA & Case report & Case study & Prospective & Outpatient & $1(100)$ & 9 \\
\hline $\begin{array}{l}\text { Mitchell et al. } \\
\text { [98] }\end{array}$ & 2017 & Australia & $\begin{array}{c}\text { Conference } \\
\text { abstract }\end{array}$ & Clinical & Prospective & $\begin{array}{l}\text { Youth at famil- } \\
\text { ial risk of BD } \\
\text { and controls }\end{array}$ & 242 (NA) & $12-30$ \\
\hline $\begin{array}{l}\text { Munhoz et al. }{ }^{\mathrm{i}} \\
\text { [99] }\end{array}$ & 2017 & Brazil & Regular article & Cohort study & Prospective & $\begin{array}{l}\text { Birth cohort } \\
\text { (Pelotas study) }\end{array}$ & $3490(48)$ & 11 \\
\hline $\begin{array}{l}\text { Özyurt et al. } \\
{[100]}\end{array}$ & 2017 & Turkey & Regular article & Clinical & Retrospective & Outpatients & $12(0)$ & $8-17$ \\
\hline $\begin{array}{l}\text { Pagliaccio et al. } \\
\text { [101] }\end{array}$ & 2017 & USA & Regular article & Clinical & Prospective & $\begin{array}{l}\text { Patients and } \\
\text { healthy con- } \\
\text { trols }\end{array}$ & $83(48)$ & $8-18$ \\
\hline $\begin{array}{l}\text { Perepletchikova } \\
\text { et al. [102] }\end{array}$ & 2017 & USA & Regular article & Clinical & Prospective & $\begin{array}{l}\text { Community and } \\
\text { outpatients }\end{array}$ & $43(44)$ & $7-12$ \\
\hline $\begin{array}{l}\text { Perhamus et al. } \\
\text { [103] }\end{array}$ & 2017 & USA & $\begin{array}{c}\text { Conference } \\
\text { abstract }\end{array}$ & Clinical & Prospective & $\begin{array}{l}\text { Patients and } \\
\text { healthy con- } \\
\text { trols }\end{array}$ & $120(45)$ & $8-18$ \\
\hline $\begin{array}{l}\text { Propper et al. } \\
\text { [104] }\end{array}$ & 2017 & Canada & Regular article & Clinical & Prospective & $\begin{array}{l}\text { Offspring of } \\
\text { parents with } \\
\text { BD or MDD }\end{array}$ & $180(53)$ & $6-18$ \\
\hline $\begin{array}{l}\text { Ramires et al. } \\
\text { [105] }\end{array}$ & 2017 & Brazil & Case report & Case study & Retrospective & Outpatients & $1(0)$ & 7 \\
\hline $\begin{array}{l}\text { Stoddard et al. }{ }^{\mathrm{e}} \\
{[106]}\end{array}$ & 2017 & USA & Regular article & Clinical & Prospective & patients & $115(44)$ & $8-17$ \\
\hline $\begin{array}{l}\text { Stoddard et al. } \\
\text { [107] }\end{array}$ & 2017 & USA & $\begin{array}{c}\text { Conference } \\
\text { abstract }\end{array}$ & Clinical & Prospective & $\begin{array}{l}\text { Patients and } \\
\text { healthy con- } \\
\text { trols }\end{array}$ & $42(42)$ & $8-21$ \\
\hline $\begin{array}{l}\text { Swetlitz et al. } \\
\text { [108] }\end{array}$ & 2017 & USA & $\begin{array}{c}\text { Conference } \\
\text { abstract }\end{array}$ & Clinical & Prospective & $\begin{array}{l}\text { Outpatients } \\
\text { and healthy } \\
\text { controls }\end{array}$ & $48(58)$ & $8-17$ \\
\hline $\begin{array}{l}\text { Taskiran et al. } \\
\text { [109] }\end{array}$ & 2017 & Turkey & $\begin{array}{c}\text { Conference } \\
\text { abstract }\end{array}$ & Clinical & Prospective & $\begin{array}{l}\text { Patients and } \\
\text { healthy con- } \\
\text { trols }\end{array}$ & 43 (NA) & M 9.5 \\
\hline $\begin{array}{l}\text { Taskiran et al. }{ }^{\mathrm{j}} \\
{[110]}\end{array}$ & 2017 & Turkey & $\begin{array}{c}\text { Conference } \\
\text { abstract }\end{array}$ & Clinical & Prospective & $\begin{array}{l}\text { Patients and } \\
\text { healthy con- } \\
\text { trols }\end{array}$ & 43 (NA) & NA \\
\hline $\begin{array}{c}\text { Tseng et al. } \\
\text { [111] }\end{array}$ & 2017 & USA & $\begin{array}{c}\text { Conference } \\
\text { abstract }\end{array}$ & Clinical & Prospective & $\begin{array}{l}\text { Patients and } \\
\text { healthy con- } \\
\text { trols }\end{array}$ & $197(59)$ & $8-18$ \\
\hline $\begin{array}{r}\text { Waxmonsky } \\
\text { et al. [112] }\end{array}$ & 2017 & USA & $\begin{array}{c}\text { Conference } \\
\text { abstract }\end{array}$ & Clinical & Retrospective & Outpatients & $56(29)$ & $7-12$ \\
\hline $\begin{array}{l}\text { Abouzed et al. } \\
\text { [113] }\end{array}$ & 2018 & Egypt & $\begin{array}{c}\text { Conference } \\
\text { abstract }\end{array}$ & Clinical & Prospective & $\begin{array}{l}\text { Offspring of } \\
\text { parents with } \\
\text { ADHD and } \\
\text { healthy con- } \\
\text { trols }\end{array}$ & 212 (NA) & $6-18$ \\
\hline $\begin{array}{c}\text { Bryant et al. } \\
{[114]}\end{array}$ & 2018 & USA & $\begin{array}{c}\text { Conference } \\
\text { abstract }\end{array}$ & Clinical & Retrospective & Patients & $360(29)$ & $4-17$ \\
\hline $\begin{array}{c}\text { Cuffe et al. } \\
\text { [115] }\end{array}$ & 2018 & USA & $\begin{array}{c}\text { Conference } \\
\text { abstract }\end{array}$ & $\begin{array}{l}\text { Population- } \\
\text { based }\end{array}$ & Prospective & $\begin{array}{l}\text { Student popula- } \\
\text { tion }\end{array}$ & $292(48)$ & $5-17$ \\
\hline $\begin{array}{l}\text { de la Peña et al. } \\
\text { [38] }\end{array}$ & 2018 & Latin America $^{1}$ & Regular article & Clinical & Prospective & Outpatients & $80(40)$ & $6-18$ \\
\hline $\begin{array}{l}\text { Delaplace et al. } \\
\text { [116] }\end{array}$ & 2018 & France & Regular article & Clinical & Prospective & Outpatients & $21(10)$ & $9-15$ \\
\hline
\end{tabular}


Table 1 (continued)

\begin{tabular}{|c|c|c|c|c|c|c|c|c|}
\hline Authors & Year & $\begin{array}{l}\text { Country of } \\
\text { origin }\end{array}$ & Article type & Study type & Study design & Sample & $N(\%$ female $)$ & Age (range) ${ }^{5}$ \\
\hline $\begin{array}{l}\text { Fridson et al. } \\
\text { [117] }\end{array}$ & 2018 & USA & $\begin{array}{c}\text { Conference } \\
\text { abstract }\end{array}$ & Clinical & Retrospective & Patients & 839 (NA) & $6-18$ \\
\hline Grau et al. [36] & 2018 & Germany & Regular article & $\begin{array}{l}\text { Population- } \\
\text { based }\end{array}$ & Prospective & Population & 2413 (NA) & $18-94$ \\
\hline $\begin{array}{l}\text { Kircanski et al. }{ }^{\mathrm{h}} \\
{[118]}\end{array}$ & 2018 & USA & Regular article & Clinical & Prospective & Community & $197(46)$ & $8-18$ \\
\hline $\begin{array}{l}\text { Miller et al. } \\
\text { [119] }\end{array}$ & 2018 & USA & Regular article & Clinical & Prospective & outpatients & $19(42)$ & $12-17$ \\
\hline $\begin{array}{c}\text { Mroczkowski } \\
\text { et al. [120] }\end{array}$ & 2018 & USA & Regular article & Juvenile justice & Retrospective & $\begin{array}{l}\text { Juvenile justice } \\
\text { involved } \\
\text { youths }\end{array}$ & $2266(30)$ & $8-18$ \\
\hline Pan et al. [121] & 2018 & Taiwan & Regular article & Clinical & Prospective & Outpatients & $58(17)$ & $7-17$ \\
\hline $\begin{array}{l}\text { Sagar-Ouriaghli } \\
\text { et al. [122] }\end{array}$ & 2018 & Great Britain & Regular article & Clinical & Prospective $^{2}$ & Outpatients & $117(\mathrm{NA})$ & $6-12$ \\
\hline $\begin{array}{l}\text { Vidal-Ribas } \\
\text { et al. [123] }\end{array}$ & 2018 & USA & Regular article & Clinical & Prospective & $\begin{array}{l}\text { Outpatients } \\
\text { and healthy } \\
\text { controls }\end{array}$ & $116(38)$ & $8-20$ \\
\hline $\begin{array}{l}\text { Walyzada et al. } \\
\text { [124] }\end{array}$ & 2018 & USA & $\begin{array}{l}\text { Conference } \\
\text { abstract }\end{array}$ & Clinical & Retrospective & Outpatients & $1088(46)$ & NA \\
\hline $\begin{array}{l}\text { Wiggins et al. } \\
\text { [125] }\end{array}$ & 2018 & USA & Regular article & Clinical & Prospective & Outpatients & $425(51)$ & $3-5$ \\
\hline $\begin{array}{l}\text { Winters et al. } \\
\text { [126] }\end{array}$ & 2018 & USA & Regular article & Clinical & Prospective & Patients & $22(31)$ & $9-15$ \\
\hline Basu et al. [127] & 2019 & Australia & Regular article & Clinical & Retrospective & Patients & $101(58)$ & $6-12$ \\
\hline $\begin{array}{l}\text { Benarous et al. } \\
\text { [128] }\end{array}$ & 2019 & France & Case report & Case study & Retrospective & Inpatients & $6(30)$ & $10-14$ \\
\hline $\begin{array}{l}\text { Benarous et al. } \\
\text { [129] }\end{array}$ & 2019 & France & $\begin{array}{c}\text { Conference } \\
\text { abstract }\end{array}$ & Clinical & Retrospective & Outpatients & $163(40)$ & $7-17$ \\
\hline Chen et al. [130] & 2019 & Taiwan & Regular article & $\begin{array}{l}\text { Population- } \\
\text { based }\end{array}$ & Prospective & Population & $4816(48)$ & $10-17$ \\
\hline $\begin{array}{c}\text { Eyre et al. }^{\mathrm{g}} \\
\text { [131] }\end{array}$ & 2019 & UK & Regular article & Clinical & Prospective & Patients & $696(16)$ & $6-18$ \\
\hline Guilé [132] & 2019 & France & $\begin{array}{l}\text { Conference } \\
\text { abstract }\end{array}$ & Clinical & Prospective & $\begin{array}{l}\text { Patients and } \\
\text { healthy con- } \\
\text { trols }\end{array}$ & $21(100)$ & $\mathrm{M} 11.7 \pm 3 \mathrm{SD}$ \\
\hline $\begin{array}{l}\text { Haller et al. } \\
\text { [133] }\end{array}$ & 2019 & USA & $\begin{array}{c}\text { Conference } \\
\text { abstract }\end{array}$ & Clinical & Prospective & $\begin{array}{l}\text { Patients and } \\
\text { healthy con- } \\
\text { trols }\end{array}$ & $44(43)$ & $8-17$ \\
\hline $\begin{array}{l}\text { Ignaszewski } \\
\text { et al. [134] }\end{array}$ & 2019 & USA & Case report & Case study & Retrospective & Outpatient & $1(0)$ & 14 \\
\hline $\begin{array}{l}\text { Linke et al. } \\
\text { [135] }\end{array}$ & 2019 & USA & Case report & Case study & Retrospective & Outpatient & $1(0)$ & 11 \\
\hline $\begin{array}{l}\text { Linke et al. } \\
{[136]}\end{array}$ & 2019 & USA & Regular article & Clinical & Prospective & $\begin{array}{l}\text { Patients and } \\
\text { healthy con- } \\
\text { trols }\end{array}$ & $118(46)$ & $11-21$ \\
\hline $\begin{array}{l}\text { Mulraney et al. } \\
\text { [137] }\end{array}$ & 2019 & Australia & $\begin{array}{c}\text { Conference } \\
\text { abstract }\end{array}$ & Cohort study & Prospective & Patients & $134(28)$ & $7-10$ \\
\hline Rice et al. [138] & 2019 & USA & Case report & Case study & Retrospective & Inpatient & $1(100)$ & 12 \\
\hline $\begin{array}{l}\text { Towbin et al. } \\
\text { [139] }\end{array}$ & 2019 & USA & Regular article & Clinical & Prospective & Patients & $53(36)$ & $7-17$ \\
\hline $\begin{array}{c}\text { Tseng et al. } \\
{[140]}\end{array}$ & 2019 & USA & Regular article & Clinical & Prospective & $\begin{array}{l}\text { Patients and } \\
\text { healthy con- } \\
\text { trols }\end{array}$ & $195(50)$ & $8-18$ \\
\hline $\begin{array}{l}\text { Tüğen et al. } \\
\text { [141] }\end{array}$ & 2019 & Turkey & Regular article & $\begin{array}{l}\text { Population- } \\
\text { based }\end{array}$ & Prospective & Community & $356(55)$ & $6-11$ \\
\hline
\end{tabular}


Table 1 (continued)

\begin{tabular}{|c|c|c|c|c|c|c|c|c|}
\hline Authors & Year & $\begin{array}{l}\text { Country of } \\
\text { origin }\end{array}$ & Article type & Study type & Study design & Sample & $N(\%$ female $)$ & Age (range) $)^{5}$ \\
\hline Ünal et al. [40] & 2010 & Turkey & Regular article & Clinical & Prospective & Outpatients & $120(49)$ & $6-17$ \\
\hline $\begin{array}{l}\text { Alexander et al. } \\
\text { [27] }\end{array}$ & 2020 & USA & Regular article & Clinical & Prospective & Community & $523(41)$ & $6-17$ \\
\hline $\begin{array}{l}\text { Benarous et al. } \\
\text { [142] }\end{array}$ & 2020 & France & Regular article & Clinical & Prospective & Patients & $30(29)$ & $6-16$ \\
\hline $\begin{array}{l}\text { Benarous et al. } \\
\text { [143] }\end{array}$ & 2020 & $\begin{array}{l}\text { France, } \\
\text { Canada }\end{array}$ & Regular article & Clinical & Retrospective & outpatients & $163(43)$ & $7-27$ \\
\hline $\begin{array}{l}\text { Chang et al. } \\
{[144]}\end{array}$ & 2020 & Taiwan & Regular article & Clinical & Prospective & Patients & $101(31)$ & $7-18$ \\
\hline $\begin{array}{l}\text { Cimino et al. } \\
\text { [145] }\end{array}$ & 2020 & Italy & Regular article & Clinical & Prospective & $\begin{array}{l}\text { Patients and } \\
\text { healthy con- } \\
\text { trols }\end{array}$ & $150(48)$ & $8-9$ \\
\hline $\begin{array}{l}\text { Haller et al. } \\
\text { [146] }\end{array}$ & 2020 & USA & $\begin{array}{l}\text { Conference } \\
\text { abstract }\end{array}$ & Clinical & Prospective & Patients & $189(34)$ & M 13.1 \\
\hline $\begin{array}{l}\text { Haller et al. } \\
\text { [147] }\end{array}$ & 2020 & USA & Regular article & Clinical & Prospective & Patients & $98(41)$ & $7-17$ \\
\hline $\begin{array}{l}\text { Johnstone et al. } \\
\text { [148] }\end{array}$ & 2020 & USA & Regular article & Clinical & Retrospective & Patients & $168(23)$ & $6-12$ \\
\hline $\begin{array}{l}\text { Laporte et al. }{ }^{\mathrm{i}} \\
\text { [45] }\end{array}$ & 2020 & Brazil & Regular article & Cohort study & Prospective & $\begin{array}{l}\text { Birth cohort } \\
\text { (Pelotas study) }\end{array}$ & 3562 (NA) & $10-12$ \\
\hline Le et al. [149] & 2020 & USA & Regular article & $\begin{array}{l}\text { Population- } \\
\text { based }\end{array}$ & Retrospective & $\begin{array}{l}\text { Patients covered } \\
\text { by Medicaid }\end{array}$ & 814,919 (49) & $<18$ \\
\hline $\begin{array}{l}\text { Tseng et al. } \\
{[150]}\end{array}$ & 2020 & USA & $\begin{array}{l}\text { Conference } \\
\text { abstract }\end{array}$ & Clinical & Prospective & Patients & 69 (NA) & M 14.5 \\
\hline
\end{tabular}

$D M D D$ disruptive mood dysregulation disorder, $A D H D$ attention deficit hyperactivity disorder, $O D D$ oppositional defiant disorder, $B D$ bipolar disorder, $S M D$ severe mood dysregulation, $M D D$ major depressive disorder. $N A$ information not available

${ }^{a}$ Mitchell et al. (2015) and (2016) report data from overlapping samples

${ }^{\mathrm{b}}$ Schipzand et al. (2015) and Mulraney et al. (2016) report data from overlapping samples

${ }^{c}$ Uran et al. (2015) abstract and regular article report on same data

${ }^{\mathrm{d}}$ Dougherty et al. (2016) and (2017) partly report on overlapping data

${ }^{\text {e}}$ Stoddard et al. (2016) and (2017) report on overlapping data

${ }^{\mathrm{f}}$ Topal et al. (2016) abstracts report data from overlapping samples

${ }^{g}$ Eyre et al. (2017) and (2019) report on overlapping data

${ }^{\mathrm{h}}$ Kircanski et al. (2017) and (2018) report on overlapping data

${ }^{i}$ Munhoz et al. (2017) and Laporte et al. (2020) report on overlapping data

${ }^{\mathrm{j}}$ Taskiran et al. (2017) abstracts report on overlapping data

${ }^{\mathrm{k}}$ Tseng et al. (2017) and (2019) report on overlapping data

${ }^{1}$ Mexico, Colombia, Chile, and Uruguay

${ }^{2}$ DMDD diagnosis was obtained retrospectively

${ }^{3}$ Where not otherwise specified, patients were in- and outpatients

${ }^{4}$ Experiment 1

${ }^{5}$ Mean (M) is given, where no information about range was available

conducted prospectively $(78.2 \% ; k=35$ abstracts, $31.8 \%)$ and $k=24$ retrospectively $(21.8 \% ; k=7$ abstracts, $6.4 \%)$. Among the prospective studies, $k=7$ assessed DMDD retrospectively $(6.4 \% ; k=1$ abstracts, $0.9 \%)$.

There was an initial increase in numbers of publications from 2013 until 2017, after which numbers dropped again: $k=1$ study in 2013 (0.9\%; $k=0$ abstracts), $k=5$ in 2014 (4.5\%; $k=0$ abstracts), $k=8$ in 2015 (7.3\%; $k=6$ abstracts,
5.5\%), $k=24$ in 2016 (21.8\%; $k=7$ abstracts, $6.4 \%), k=29$ in $2017(26.4 \% ; k=16$ abstracts, $14.5 \%), k=16$ in 2018 (14.5\%; $k=5$ abstracts, $4.5 \%)$, and $k=16$ in 2019 (14.5\%; $k=4$ abstracts, $3.6 \%)$ and $k=11$ in $2020(10.0 \% ; k=2$ abstracts, $1.8 \%$ ).

Most of the included studies stem from the United States of America $(k=66,60.0 \% ; k=26$ abstracts, $23.6 \%)$. Other countries of origin include Turkey $(k=12,10.9 \% ; k=6$ 
abstracts, 5.5\%), France $(k=6,5.5 \% ; k=2$ abstracts, $1.8 \%)$, Australia $(k=5,4.5 \% ; k=3$ abstracts, $2.7 \%)$, Brazil $(k=3$, $2.7 \% ; k=0$ abstracts), Canada $(k=3,2.7 \% ; k=1$ abstracts $)$, United Kingdom $(k=3,2.7 \% ; k=0$ abstracts), Taiwan $(k=3$, $2.7 \% ; k=0$ abstracts), India $(k=2,1.8 \% ; k=1$ abstract $)$, Spain $(k=2,1.8 \% ; k=1$ abstract), Italy $(k=2,1.8 \% ; k=0$ abstracts), Egypt ( $k=1,0.9 \% ; k=1$ abstract) and Germany ( $k=1,0.9 \% ; k=0$ abstracts). $K=1$ regular article includes data from Mexico, Colombia, Chile, and Uruguay (0.9\%).

Most study samples consisted of patients (in- and/or outpatients) $k=85$ (77.3\%; $k=33$ abstracts, $30.0 \%)$. Of those, some reported to include only outpatients $(n=39$, $35.5 \% ; k=10$ abstracts, $9.1 \%$ ), or inpatients $(n=7,6.4 \%$; $k=3$ abstracts, $2.7 \%$ ). Further, study samples consisted of community ( $n=10,9.1 \% ; k=2$ abstracts, $1.8 \%$ ), population ( $n=7,6.4 \% ; k=2$ abstracts, $1.8 \%)$, youth at familial risk of psychiatric disorders $(n=4,3.6 \% ; k=3$ abstracts, $2.7 \%$ ) birth cohorts ( $n=2,1.8 \% ; k=0$ abstracts), juvenile justice involved youths ( $n=1,0.9 \% ; k=0$ abstracts), and overweight patients ( $n=1,0.9 \%, k=1$ abstract). Many of the studies further examined healthy controls in addition to a patient group $(n=26,23.6 \%)$. Sample sizes ranged from $k=1$ in case-reports to $k=6483$ in a large population-based study. Examined ages lay between 2 and 94 years of age, while most samples' ages ranged from early school-age to adolescence or young adulthood.

\section{Measurement of DMDD diagnosis}

A variety of instruments were used to diagnose DMDD in the included studies. The instrument used most often was the Kiddie Schedule for Affective Disorders and Schizophrenia Present and Lifetime Version, K-SADS-PL [19] ( $n=48,43.6 \% ; k=20$ abstracts, $18.2 \%$ ) in combination with the DMDD module (Table 2), $k=27$ (24.5\%; $k=12$ abstracts, 10.9\%). The Preschool Age Psychiatric Assessment, PAPA [20] was used in $k=7$ studies $(6.4 \% ; k=1$ abstracts, $0.9 \%$ ), of which $k=4$ did so in combination with ODD and depression sections. In $k=3$ (2.7\%) studies each, the Child and Adolescent Psychiatric Assessment, CAPA [21] ( $n=0$ abstracts), the Diagnostic Interview Schedule for Children, Version IV, DISC-IV [22] ( $n=1$ abstract, 0.9\%), and the Washington University in St. Louis Kiddie Schedule for Affective Disorders and Schizophrenia, WASH-U-KSADS [23] ( $n=1$ abstract, $0.9 \%)$ were used. In $k=2$ studies $(1.8 \%)$ each, the Breton, Bergeron and Labelle DMDD Scale [24] ( $n=1$ abstract, $0.9 \%$ ), the Conners rating scales [25] ( $n=1$ abstract, 0.9\%), the Development and Well-Being Assessment, DAWBA [26] and the Extended Strengths and Weaknesses Assessment of Normal Behavior, E-SWAN [27] ( $n=1$ abstracts, $0.9 \%$ ) were used. Instruments used in $k=1$ (0.9\%) regular articles each included the Child and Adolescent Symptom Inventory, CASI [28], the Child Behavior
Check List dysregulation profile, CBCL-DP [29], the Children's Interview for Psychiatric Syndromes, ChIPS [30] in combination with the Mini-International Neuropsychiatric Interview for Children and Adolescents, MINI-KID [31], the Composite International Diagnostic Interview CIDI [32], the Diagnostic Infant and Preschool Assessment, DIPA [33], the Mandarin Version of the K-SADS-Epidemiological Version for DSM-5, K-SADS-E [34], the Structured Clinical Interview for DSM-IV, SCID-IV [35], a self-created set of six questions [36], and the Voice Diagnostic Interview Schedule for Children, V-DISC [22]. A not otherwise specified structured interview was reported in $k=1$ conference abstract [37].

In $k=8$ studies $(7.3 \% ; k=2$ abstracts, $1.8 \%)$ a clinical diagnosis was made without any specific measures and in $k=7$ studies $(6.4 \% ; k=3$ abstracts, $2.7 \%$ ) diagnosis was made using chart review or Medicaid records $(n=1)$. Finally, $k=10(9.1 \% ; k=6$ abstracts, $5.5 \%)$ studies did not provide any information on the measure used.

In most of the measures used in the included studies, a clinician rated the patients' and participants' statements and behavior $(n=91,82.7 \%)$, while others consisted of a parent$(n=3,2.7 \%)$, or self-rating $(n=4,3.6 \%)$. No information about the rater was given in $k=10(9.1 \%)$ studies.

\section{Psychometric properties}

In $k=79$ studies $(71.8 \% ; k=17$ abstracts, $15.5 \%$ ), any information on the presence or absence of psychometric properties of the measure used to diagnose DMDD was given or obtained from the authors. Of those, in $k=39(35.5 \%$; $k=4$ abstracts, $3.6 \%$ ) no psychometric properties have been obtained or reported as part of the study or using the study data. In the remaining $k=40$ studies $(36.4 \%, k=13$ abstracts, $11.8 \%$ ), the most commonly reported psychometric property was reliability, with $k=33(30.0 \% ; k=13$ abstracts, $11.8 \%)$ reporting inter-rater reliability ranging from $\kappa=0.6$ to 1 and $k=29$ (26.4\%; $k=11$ abstracts, $10.0 \%)$ reporting intraclass correlation coefficients. Three studies assessed internal consistency with Cronbach's alpha $=0.92$ for a Spanish version of the K-SADS-PL modified under the DSM-5 to diagnose DMDD [38], and Cronbach's alpha $=0.75$ for the PAPA [39] and 0.98 for the E-SWAN DMDD scale [27]. In the studies of the NIMH group around Dr. Ellen Leibenluft $(n=25,22.7 \%)$, raters were trained to reach inter-rater reliability with $\kappa \geq 0.9$, before they contributed to interviews/ data collection for the respective studies. Cases were further discussed in conference with other reliable clinicians and in a lab meeting where leading clinicians reviewed the core criteria before diagnosis was made. The same group also provided ICCs $\geq 0.9$ differentiating the DMDD module from the mania/hypomania part of the K-SADS-PL. One study examined consensus validity between a clinical psychiatric 


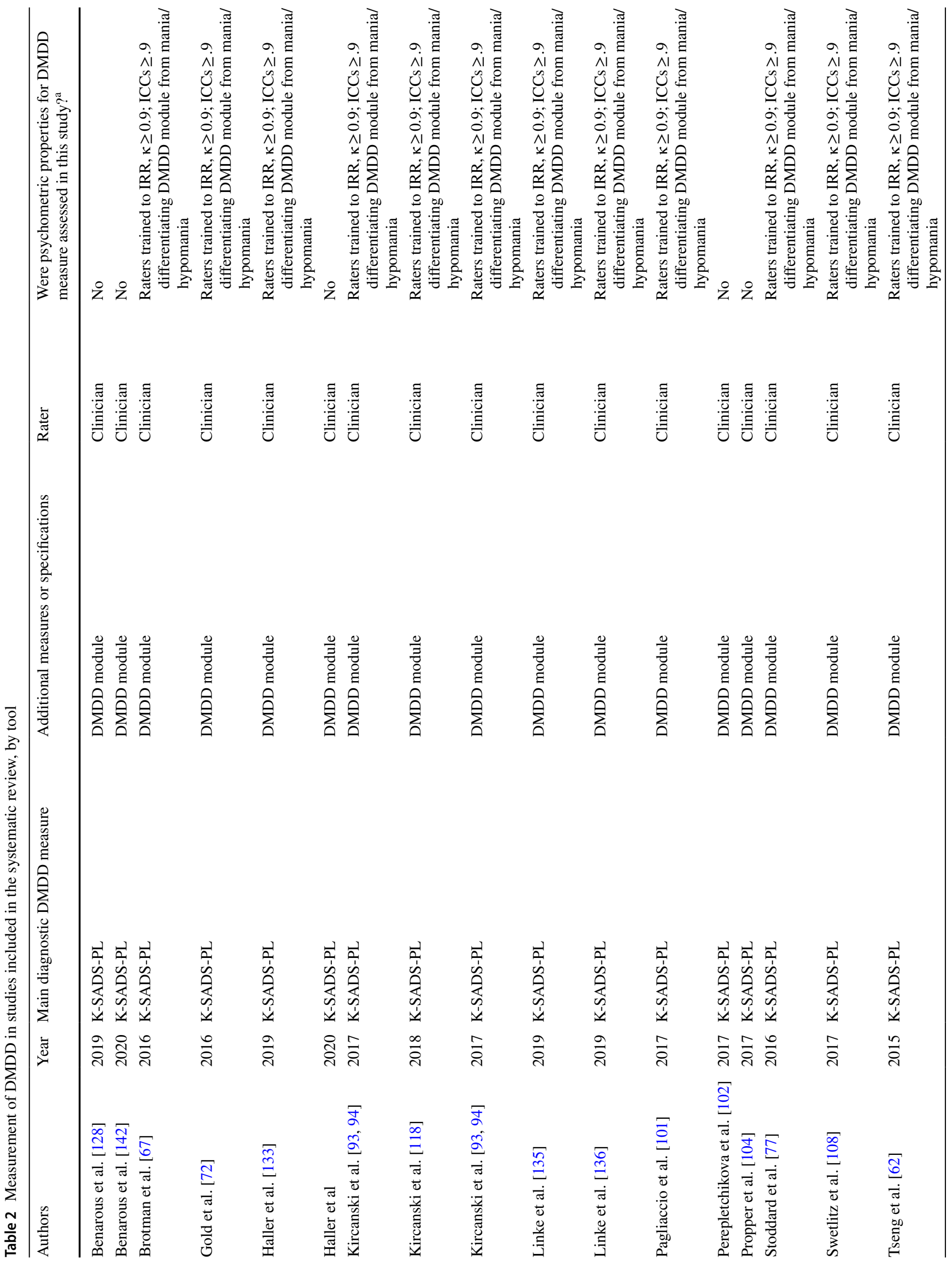




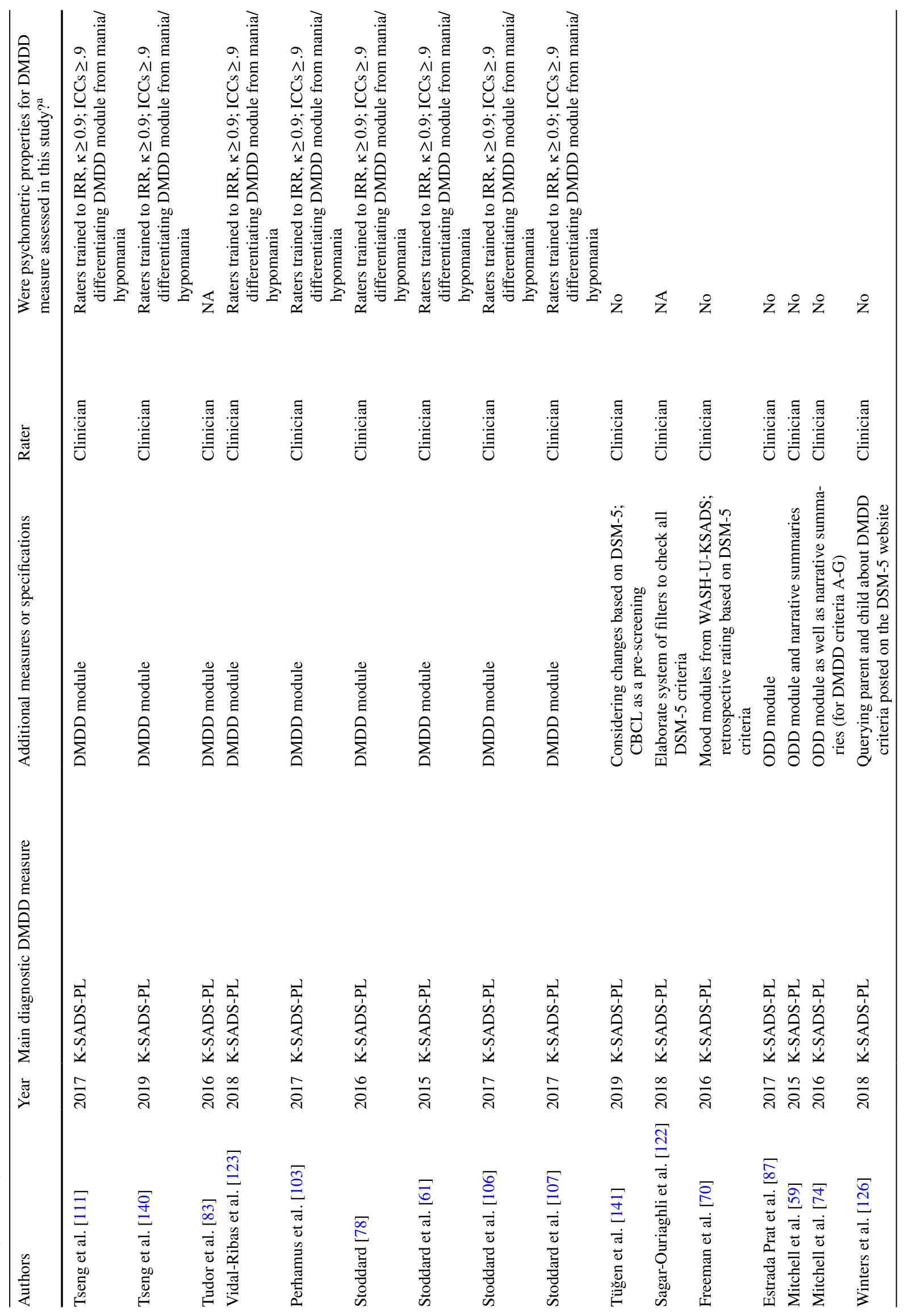




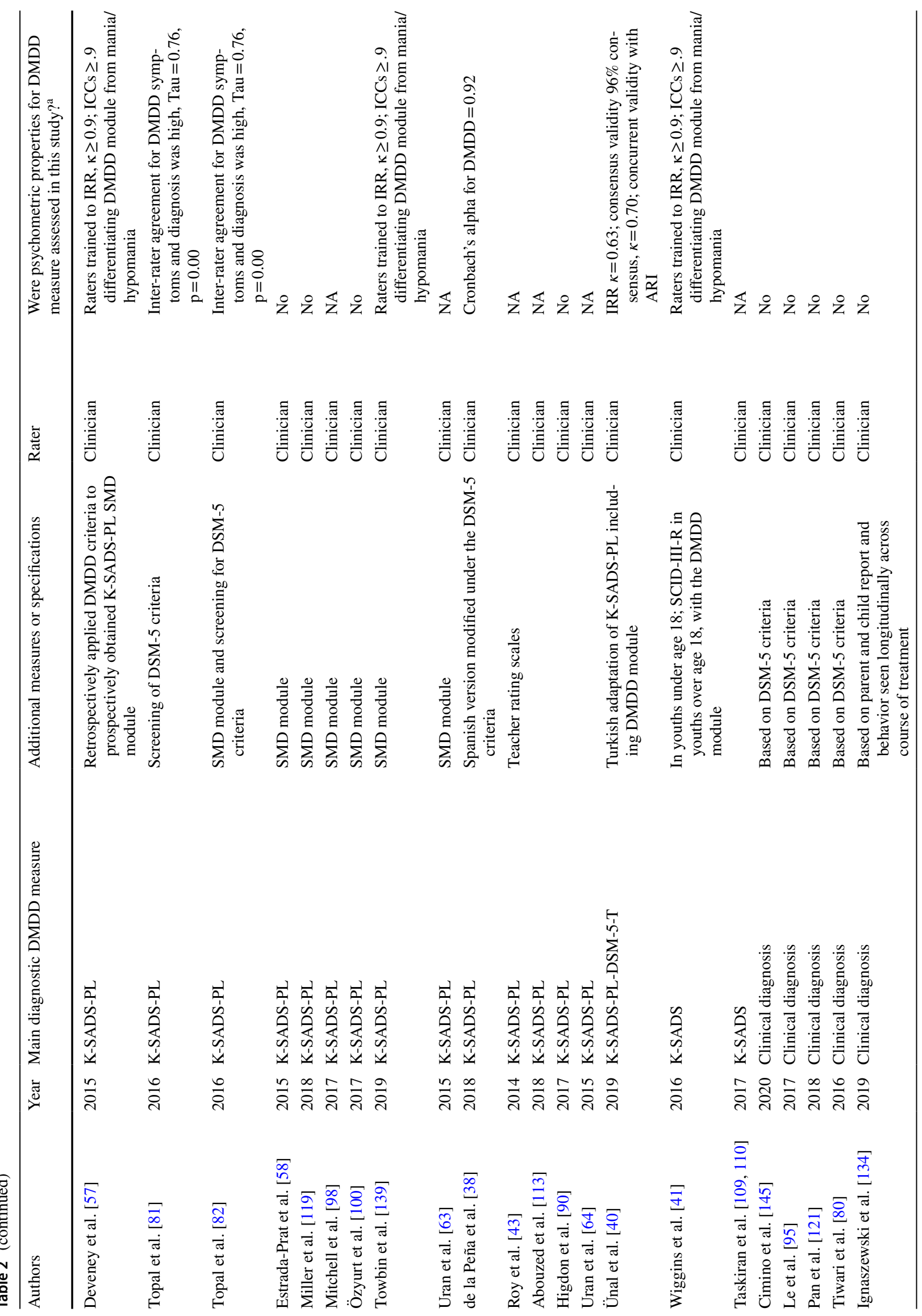




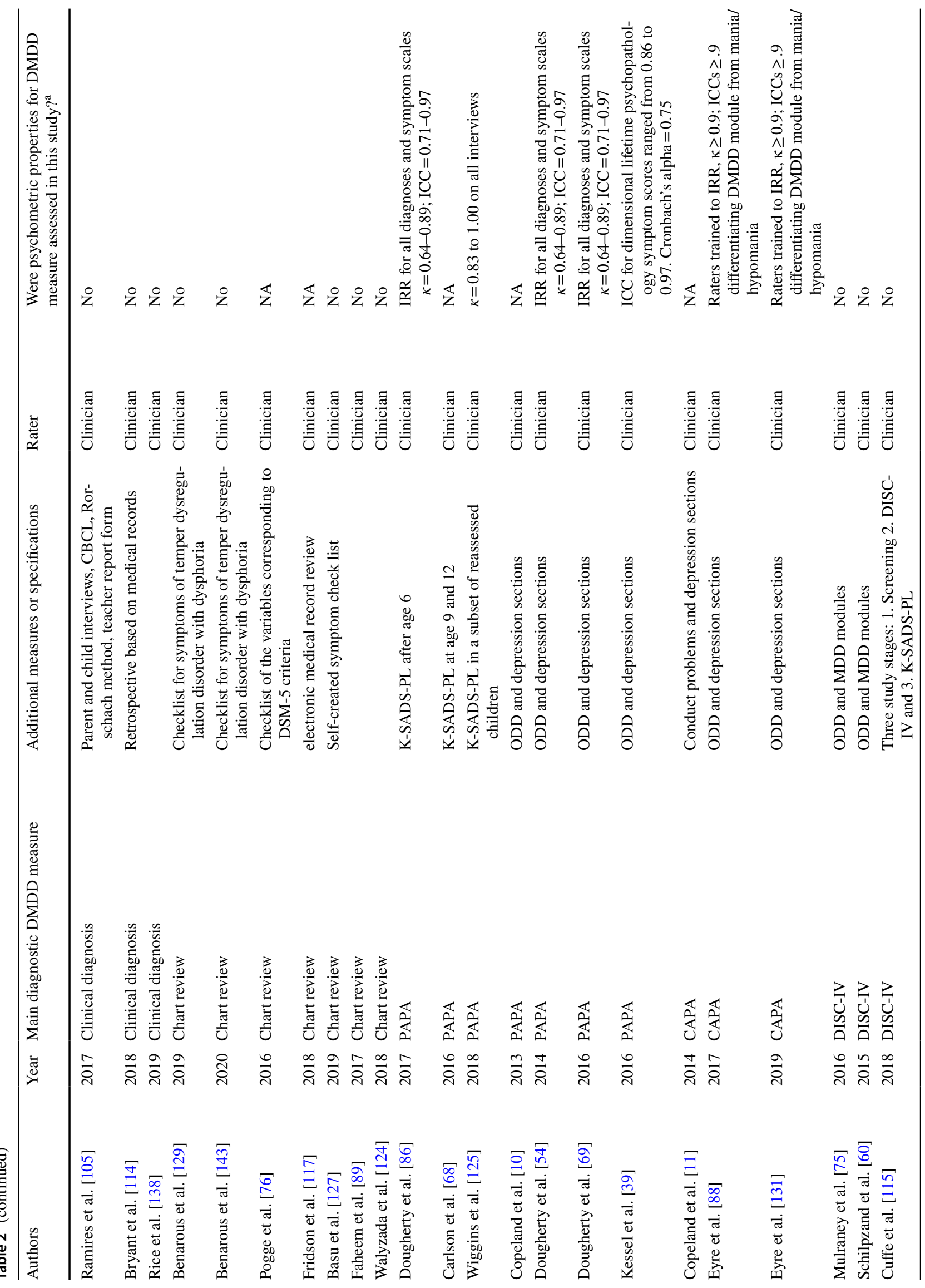




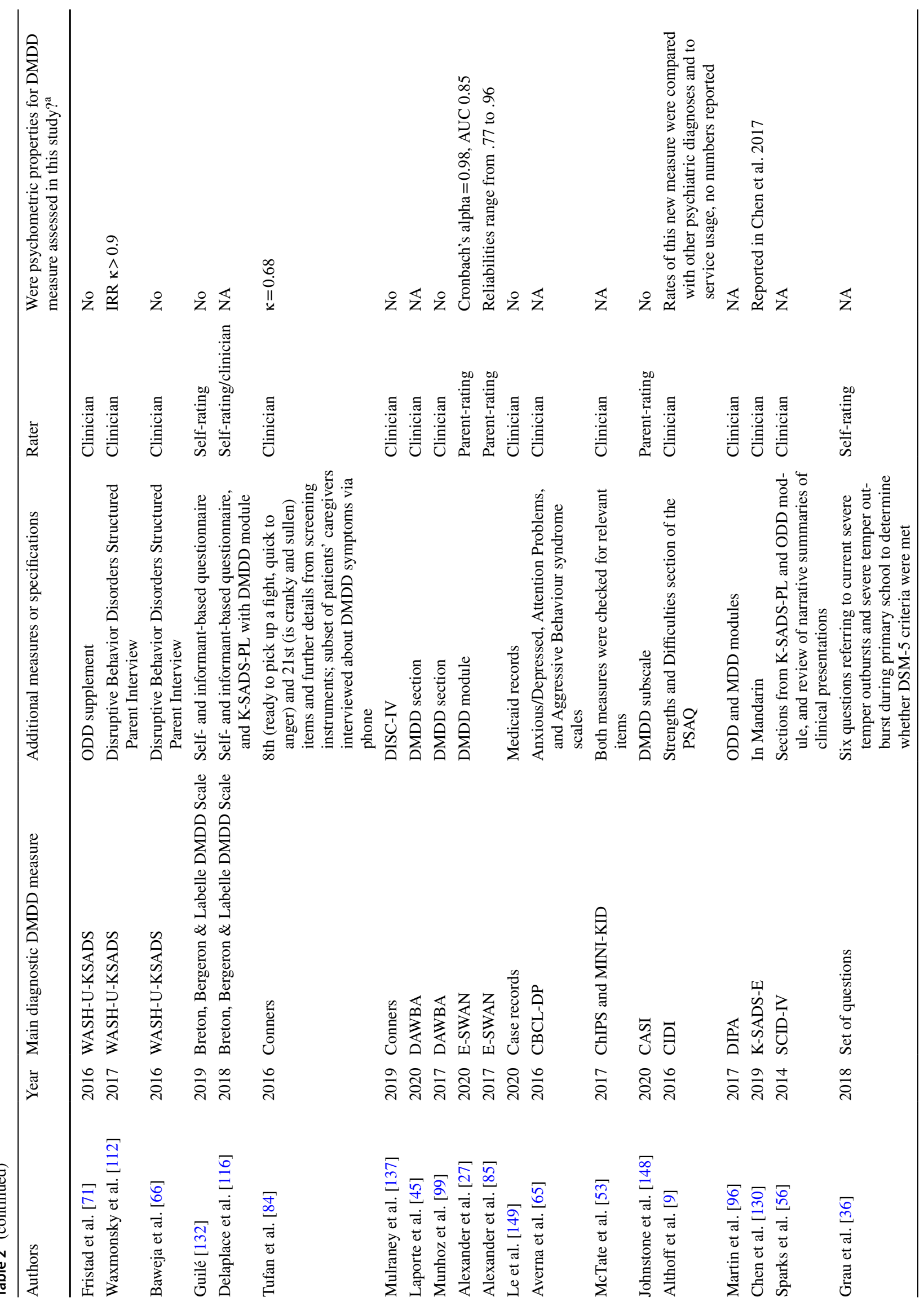


interview based on DSM-5 diagnostic criteria and the Turkish version of the DSM-5 version of the K-SADS-PL (K-SADS-PL-DSM-5-T), led by two independent clinicianresearchers [40]. A consensus of $96 \%, \kappa=0.63$ was reached. Further, concurrent validity was evaluated with the Affective Reactivity Index (ARI), $\kappa=0.70$. One study generated Receiver Operating Characteristic (ROC) curves to obtain Area Under the Curve (AUC) for their diagnostic instrument, as a measure of predictive validity. With an AUC value of 0.85 , the E-SWAN DMDD scale performed equally well in predicting diagnoses compared to the Affective Reactivity Index [27].

\section{Discussion}

Evidence from this systematic review points to a variety of different measures used for the evaluation and diagnosis of DMDD. The majority of studies used clinician-rated structured interviews in combination with DMDD specific symptom checklists. Few studies employed questionnaires or interviews specifically designed to measure DMDD or its severity. In the following, some of the most used measures are presented in more detail, before practical aspects, such as available languages and cost as well as diagnostic challenges and future directions are discussed.

By far the most often used instrument was the K-SADSPL in combination with the DMDD module. The K-SADS$\mathrm{PL}$ is a semi-structured interview to diagnose mental disorders in children aged 6-18. Administration time is estimated to be about $75 \mathrm{~min}$ for psychiatric patients and $35-45 \mathrm{~min}$ for healthy control subjects. It is freely available for download online. It has high inter-rater reliability and good to excellent test-retest reliability [19]. The DMDD module has been developed by a workgroup around Leibenluft, in collaboration with the K-SADS developer Kaufman. A prior version of this module was based on a research diagnosis coined severe mood dysregulation (SMD) [4]. The DMDD module is a checklist consisting of four items probing for the DSM-5 criteria to be met (Fig. 2, see supplementary material for the DSM-5 diagnostic criteria A-K). With training and case discussion, the module can be administered with high inter-rater reliability [41]. It has further shown to differentiate well between other mood disorders such as mania/ hypomania.

Our study's findings revealed different methodological approaches to diagnosing DMDD. Some of the instruments utilized in the reviewed studies consisted of a symptom checklist. This was the case not only for the K-SADS-PL DMDD module but also for its precursor, the SMD module or the ODD module. While the checklist format might suggest simplicity, it is most often used in the context of the more comprehensive K-SADS-PL semi-structured interview, 
1. Criterion A-D have been present for 12 months or more, no period of three or more consecutive months without symptoms.

2. Criterion A-D are present in at least two of the three settings listed below:

Specify: Home School Peers

3. Onset of Criterion A-E before age of 10 .

4. Evidence of Disruptive Mood Dysregulation Disorder

Fig. 2 K-SADS-PL DMDD module. Each of the questions are evaluated with 0,1 or 2 for current and/or past episodes. The diagnostic criteria of DMDD are listed below the questions in the module (see

which is used by raters to create a proxy diagnostic using a combination of ODD, depression, or mania criteria, and thereby empirically derive a DMDD diagnosis. Moreover, a combination of comprehensive structured or semi-structured interviews (e.g., K-SADS-PL, SCID, DISC or CIDI) and self-made checklists or clinical evaluation to probe for DSM criteria have been employed. An approach that has further been adopted in some of the reviewed studies was to search established interviews or questionnaires (CBCL-DP, Conners, ChIPS, MINI or PAPA/CAPA/DIPA) for items relevant to the DMDD diagnosis. This approach likely stems from the fact that these studies assessed DMDD retrospectively in data not collected with the focus of determining the prevalence of DMDD.

Few instruments have been deliberately designed to diagnose DMDD. Those identified by this systematic review were the K-SADS-PL DMDD module, the Breton, Bergeron and Labelle DMDD scale (available as a semi-structured interview and questionnaire), the E-SWAN DMDD module (interview) and the DAWBA DMDD section (interview; see Table 3 for an overview of instruments designed to diagnose DMDD). The instruments contain 4-34 items assessing occurrences, frequencies, and circumstances of temper tantrums/outbursts and irritable or angry mood. All instruments are available in the English language. The Breton, Bergeron supplementary material for the DSM-5 diagnostic criteria). Reprint authorized by Joan Kaufman, owner of the copyright of the K-SADSPL

and Labelle DMDD Scale is additionally available in French, and the DAWBA DMDD section additionally exists in Danish and Portuguese. The E-SWAN and DAWBA scales are freely available online or upon request to the authors. Indicated age ranges are similar, encompassing preschool age to early adulthood. While the K-SADS-PL DMDD module, the Breton, Bergeron and Labelle DMDD Scale, and the DAWBA DMDD section provide categorical outcomes, the E-SWAN DMDD module is designed to capture DMDD symptoms dimensionally. This scale reconceptualizes each diagnostic criterion for DMDD as a behavior, which can range from high (strengths) to low (weaknesses). Regarding the psychometric properties, it seems that the DMDD module has been evaluated most often, as high levels of reliability are reported in many studies. However, these reliabilities have been reached artificially by training raters to differentiate K-SADS-PL DMDD from mania modules. Although useful for the clinic, this approach does not correspond to the evaluation of reliability as a measure of consistency between raters for a certain diagnostic instrument used in a study. Therefore, a more comprehensive psychometric evaluation of this widely used measure is necessary. Besides the DMDD module, psychometric properties have been reported for the E-SWAN DMDD module. The reliability of this scale has been reported to be excellent (Cronbach's

Table 3 Instruments designed to diagnose DMDD

\begin{tabular}{|c|c|c|c|c|c|c|}
\hline & Method & $\begin{array}{l}\text { Number of } \\
\text { items }\end{array}$ & $\begin{array}{l}\text { Freely avail- } \\
\text { able/costs }\end{array}$ & Languages available & $\begin{array}{l}\text { Outcome } \\
\text { dimensional }\end{array}$ & $\begin{array}{l}\text { Indicated } \\
\text { age range }\end{array}$ \\
\hline K-SADS-PL DMDD module & Symptom Checklist & 4 & Yes & English & No & $6-19$ \\
\hline $\begin{array}{l}\text { Breton, Bergeron \& Labelle } \\
\text { DMDD Scale }\end{array}$ & $\begin{array}{l}\text { Semi-structured inter- } \\
\text { view/questionnaire }\end{array}$ & 11 & NA & English, French & No & NA \\
\hline DAWBA DMDD section & Interview & 34 & Yes & English, Danish, Portugese & No & $5-18$ \\
\hline E-SWAN DMDD module & Interview & 10 & Yes & English & Yes & $6-17$ \\
\hline
\end{tabular}

$D M D D$ disruptive mood dysregulation disorder, $N A$ information not available 
alpha $=0.98$ ). Reporting of psychometric properties of the other DMDD scales is still pending. Studies using tools to diagnose DMDD followed a broad spectrum of study objectives and hypotheses. Thus, the DMDD measure and its psychometric properties might not have been the focus of attention, which might be the reason for not providing this information. However, to determine gold-standard measurement, psychometric evaluation of the currently used diagnostic measures is necessary.

When assessing the psychometric properties of the instruments used in the included studies, mainly measures of reliability have been considered and reported. However, the psychometric evaluation of a diagnostic tool ideally also contains the assessment of its validity. Neither contentrelated (e.g., construct validity, factorial structure) nor criterion-related types of validity (e.g., concurrent or predictive validity) have been considered broadly in existing studies. One study reported substantial consensus validity $(\kappa=0.63)$ and concurrent validity $(\kappa=0.70)$ of a Turkish version of the K-SADS-PL [40]. A further study showed substantial predictive validity of the E-SWAN DMDD module $(\mathrm{AUC}=0.85)$ [27]. Consequently, measures of validity require more attention in future research on the measurement of DMDD and should guide the reporting of respective measures in future studies.

Given the aim of the present systematic review, to provide an overview of existing instruments for the assessment of DMDD and their use in the diagnostic process, we refrained from conducting a formal risk of bias assessment of included studies. The potential risk of bias does not interfere with the aim of the present review and was thus deemed irrelevant.

Since the advent of DMDD, clinicians and researchers have noted various challenges and the diagnosis is not without controversy [17]. The characteristic symptoms of DMDD, namely irritable mood and temper outbursts are observed across multiple disruptive behavior and mood disorders and the validity of DMDD as a distinct diagnosis has been questioned [13, 42, 43]. Further, DMDD could not be distinguished from ODD based on symptomatology alone in a population-based study [44]. It has further been criticized that alternative thresholds for defining DMDD, as well as a closer investigation of clinically relevant thresholds, have so far only partly been considered in the existing literature [45]. The lack of precision in diagnosing DMDD might in part account for the criticism voiced about the clinical entity of DMDD. Similarly, the heterogeneity in measurement of DMDD up to date, as found in the present systematic review of the literature, might account for variations in current prevalence and comorbidity rates as well as findings on associations with risk factors or functional outcomes in individuals with DMDD. Studies designed a priori with appropriate instruments to capture DMDD are therefore necessary [46].
While the diagnostic entity of DMDD may be a useful clinical heuristic, many researcher-clinicians focus their efforts on broader transdiagnostic constructs, such as irritability [8]. Irritability has been defined as a heightened proneness to anger relative to peers $[47,48]$ which can be seen as a personality trait with a continuous distribution across the population. In children and adolescents with DMDD, by definition, irritability is severe and expressed stably across time. In the last decade, there has been a marked increase in irritability research and there have been neuroscientific as well as treatment-related approaches to understanding pathophysiological mechanisms [41, 49]. Until now, whether persistent irritability between temper outbursts and the outbursts themselves are independent of each other, or whether the mood between outbursts is rather a concatenation of less severe tantrums, remains unknown.

In addition to further psychometric evaluation of current diagnostic measures and the development of a gold-standard diagnostic measure, adjuvant measurement approaches have become popular in the last decade. One promising approach to describe the full spectrum of irritability and temper outbursts in patients' everyday lives is ecological momentary assessment (EMA; also known as experience sampling method or ambulatory assessment). This involves the repeated sampling of patients' experiences or mood, performed via a handheld device such as a mobile phone. This measurement method has high ecological validity, avoiding biases due to retrospective assessments [50]. The repeated measurement of affect, with multiple measurements during the day over several days, potentially in children or their parents might be insightful in the characterization of hourly and daily fluctuations of mood in patients with irritability and/or DMDD.

To inform the debate around the diagnostic entity of DMDD, the application of Research Domain Criteria (RDoC) constructs may yield greater clarity in terms of underlying processes and thus inform nosology as well as appropriate interventions [51]. The constructs of frustrative non-reward (Negative Valence Domain), reward prediction error (Positive Valence domain), attention and language (Cognitive domain) as well as arousal (Arousal and Regulatory systems) have been found to be particularly promising in this regard.

\section{Limitations of the review}

The present systematic review encompasses literature involving instruments for the categorical diagnosis of DMDD. In view of the described developments regarding dimensional aspects of DMDD, a systematic review of the literature on dimensional constructs, such as irritability would be informative and topical. Similarly, a comprehensive overview on 
the examination of developmentally non-appropriate temper tantrums would be of interest in this regard.

A substantial proportion of the studies included in this systematic review stems from one laboratory in the United States. More studies evaluating the reliability and validity of the DMDD diagnosis should be conducted in other laboratories, to reduce the potential bias of findings and address cultural differences.

Psychological assessment should not be made based on any one instrument in isolation. Rather, test findings should be integrated with information from personal and educational histories and in collaboration with other clinicians $[52,53]$. Consequently, using any current instruments to evaluate DMDD will require additional query and clinical evaluation. For research purposes, however, standardized assessment methods are inevitable.

\section{Conclusion and future directions}

A variety of different measures have been used for the evaluation of DMDD. The most commonly used and established instrument consists of a symptom checklist, while more recently developed structured interviews and questionnaires are still to establish their reliability and validity in diagnosing DMDD. Dimensional and experimental approaches to assessing irritability and temper outbursts as well as their interrelation might bring forth more clarity about DMDD symptomatology in children.

Supplementary Information The online version contains supplementary material available at https://doi.org/10.1007/s00787-021-01840-4.

Acknowledgements We would like to thank all authors who responded to our requests and sent us additional information on their studies for the purpose of this systematic review of the literature (in alphabetical order by last name): Althoff Robert R., Basu Soumya, Baweja Raman, Benarous Xavier, Brotman Melissa A., Bryant Beverly J., Carlson Gabrielle A., Cimino Silvia, Coffey Barbara J, Copeland William E., Corell Christoph U., Cruz Maria, Cuffe Steven P., Davis Winders Deborah, Deveney Christen M., Dougherty Lea R., Estrada Prat Xavier, Eyre Olga, Freeman Andrew J., Fristad Mary A., Gold Andrea L., Guilé Jean-Marc, Haller Simone P., Higdon Claudine, Hulvershorn Leslie A., Ignaszewski Martha J., Kircanski Katharina, Labelle Réal, Linke Julia O., McTate Emily A., Miller Leslie, Mitchell Rachel H., Mroczkowski Megan M., Mulraney Melissa, Munhoz Tiago Neuenfeld, Özyurt Gonca, Pagliaccio David, Perepletchikova Franchesca, Perhamus Gretchen, Pogge David L., Ramires Vera R., Rohde Luis A., Santosh Paramala, Stoddard Joel, Stringaris Argyris, Swetlitz Caroline, Topal Zehra, Tseng Wan-Ling, Tudor Megan E., Tüğen Leyla E., Uher Rudolf, Ulloa Elena, Vidal-Ribas Pablo, Walyzada Frozan, Waxmonsky James G., Wiggins Jillian L., Zeni Cristian P. We would also like to thank our research assistant Laura Auderset for her great help in the study selection process.

Author contributions IM-L and JK conceptualized the study, IM-L performed the literature search and data analysis and drafted the work, IM-L, JK and MK critically revised the work.
Funding Open Access funding provided by Universität Bern. Institutional funding from Child and Adolescent Psychiatry and Psychotherapy, University of Bern, Switzerland.

Availability of data and material Can be requested from the corresponding author.

Code availability Not applicable.

\section{Declarations}

Conflict of interest All authors declare that they have no conflict of interest.

Open Access This article is licensed under a Creative Commons Attribution 4.0 International License, which permits use, sharing, adaptation, distribution and reproduction in any medium or format, as long as you give appropriate credit to the original author(s) and the source, provide a link to the Creative Commons licence, and indicate if changes were made. The images or other third party material in this article are included in the article's Creative Commons licence, unless indicated otherwise in a credit line to the material. If material is not included in the article's Creative Commons licence and your intended use is not permitted by statutory regulation or exceeds the permitted use, you will need to obtain permission directly from the copyright holder. To view a copy of this licence, visit http://creativecommons.org/licenses/by/4.0/.

\section{References}

1. American Psychiatric Association (2013) Diagnostic and statistical manual of mental disorders, 5th edn. American Psychiatric Association, Washington, DC

2. Leibenluft E (2011) Severe mood dysregulation, irritability, and the diagnostic boundaries of bipolar disorder in youths. Am J Psychiatry 168:129-142. https://doi.org/10.1176/appi.ajp.2010. 10050766

3. Stringaris A, Vidal-Ribas P, Brotman MA, Leibenluft E (2018) Practitioner review: definition, recognition, and treatment challenges of irritability in young people. J Child Psychol Psychiatry 59:721-739. https://doi.org/10.1111/jcpp.12823

4. Leibenluft E, Charney DS, Towbin KE et al (2003) Defining clinical phenotypes of juvenile mania. Am J Psychiatry 160:430437. https://doi.org/10.1176/appi.ajp.160.3.430

5. Adleman NE, Kayser R, Dickstein D et al (2011) Neural correlates of reversal learning in severe mood dysregulation and pediatric bipolar disorder. J Am Acad Child Adolesc Psychiatry 50:1173-1185.e2. https://doi.org/10.1016/j.jaac.2011.07.011

6. Deveney CM, Connolly ME, Jenkins SE et al (2012) Neural recruitment during failed motor inhibition differentiates youths with bipolar disorder and severe mood dysregulation. Biol Psychol 89:148-155. https://doi.org/10.1016/j.biopsycho.2011.10. 003

7. Thomas LA, Brotman MA, Muhrer EJ et al (2012) Parametric modulation of neural activity by emotion in youth with bipolar disorder, youth with severe mood dysregulation, and healthy volunteers. Arch Gen Psychiatry 69:1257-1266. https://doi.org/10. 1001/archgenpsychiatry.2012.913

8. Brotman MA, Kircanski K, Stringaris A et al (2017) Irritability in youths: a translational model. Am J Psychiatry 174:520-532. https://doi.org/10.1176/appi.ajp.2016.16070839 
9. Althoff RR, Crehan ET, He J-P et al (2016) Disruptive mood dysregulation disorder at ages 13-18: results from the National Comorbidity Survey-Adolescent Supplement. J Child Adolesc Psychopharmacol 26:107-113. https://doi.org/10.1089/cap.2015. 0038

10. Copeland WE, Angold A, Costello EJ, Egger H (2013) Prevalence, comorbidity, and correlates of DSM-5 proposed disruptive mood dysregulation disorder. Am J Psychiatry 170:173-179. https://doi.org/10.1176/appi.ajp.2012.12010132

11. Copeland WE, Shanahan L, Egger H et al (2014) Adult diagnostic and functional outcomes of DSM-5 disruptive mood dysregulation disorder. Am J Psychiatry 171:668-674. https://doi.org/10. 1176/appi.ajp.2014.13091213

12. Tapia V, John RM (2018) Disruptive mood dysregulation disorder. J Nurse Pract 14:573-578.e3. https://doi.org/10.1016/j. nurpra.2018.07.007

13. Axelson D, Findling RL, Fristad MA et al (2012) Examining the proposed disruptive mood dysregulation disorder diagnosis in children in the longitudinal assessment of manic symptoms study. J Clin Psychiatry 73:1342-1350. https://doi.org/10.4088/ JCP.12m07674

14. Mayes SD, Waxmonsky J, Calhoun SL et al (2015) Disruptive mood dysregulation disorder (DMDD) symptoms in children with autism, ADHD, and neurotypical development and impact of co-occurring ODD, depression, and anxiety. Res Autism Spectr Disord 18:64-72. https://doi.org/10.1016/j.rasd.2015.07. 003

15. Mayes SD, Waxmonsky JD, Calhoun SL, Bixler EO (2016) Disruptive mood dysregulation disorder symptoms and association with oppositional defiant and other disorders in a general population child sample. J Child Adolesc Psychopharmacol 26:101-106. https://doi.org/10.1089/cap.2015.0074

16. De Rosa C (2018) ICD-11 sessions in the 17th World Congress of Psychiatry. World Psychiatry. 17(1):119-120. https://doi.org/ 10.1002/wps.20507

17. Bruno A, Celebre L, Torre G et al (2019) Focus on disruptive mood dysregulation disorder: a review of the literature. Psychiatry Res 279:323-330. https://doi.org/10.1016/j.psychres.2019.05. 043

18. Moher D, Liberati A, Tetzlaff J et al (2009) Preferred reporting items for systematic reviews and meta-analyses: the PRISMA statement. PLoS Med 6:e1000097. https://doi.org/10.1371/journ al.pmed.1000097

19. Kaufman J, Birmaher B, Brent D et al (1997) Schedule for affective disorders and schizophrenia for school-age children-present and lifetime version (K-SADS-PL): initial reliability and validity data. J Am Acad Child Adolesc Psychiatry 36:980-988. https:// doi.org/10.1097/00004583-199707000-00021

20. Egger H, Angold A (2004) The preschool age psychiatric assessment (PAPA): a structured parent interview for diagnosing psychiatric disorders in preschool children. In: DelCarmen-Wiggins R, Carter A (eds) Handbook of Infant and Toddler Mental Health Assessment. Oxford University Press, New York, pp 223-243

21. Angold A, Costello EJ (2000) The Child and Adolescent Psychiatric Assessment (CAPA). J Am Acad Child Adolesc Psychiatry 39:39-48. https://doi.org/10.1097/00004583-200001000-00015

22. Shaffer D, Fisher P, Lucas CP et al (2000) NIMH Diagnostic Interview Schedule for Children Version IV (NIMH DISC-IV): description, differences from previous versions, and reliability of some common diagnoses. J Am Acad Child Adolesc Psychiatry 39:28-38. https://doi.org/10.1097/00004583-200001000-00014

23. Geller B, Zimerman B, Williams M et al (2001) Reliability of the Washington University in St. Louis Kiddie Schedule for Affective Disorders and Schizophrenia (WASH-U-KSADS) Mania and Rapid Cycling Sections. J Am Acad Child Adolesc
Psychiatry 40:450-455. https://doi.org/10.1097/00004583-20010 4000-00014

24. Boudjerida A, Labelle R, Bergeron L et al (2018) Disruptive mood dysregulation disorder scale in adolescence. 23rd World Congress of the International Association for Child \& Adolescent Psychiatry and Allied Professions, Prague

25. Conners CK (2008) Conners 3rd edition manual. In: Multi-health systems, Toronto Ontario, Canada

26. Goodman R, Ford T, Richards H et al (2000) The development and well-being assessment: description and initial validation of an integrated assessement of child and adolescent psychopathology. J Child Psychol Psychiatry 41:645-655. https://doi.org/10. 1017/S0021963099005909

27. Alexander LM, Salum GA, Swanson JM, Milham MP (2020) Measuring strengths and weaknesses in dimensional psychiatry. J Child Psychol Psychiatr 61:40-50. https://doi.org/10.1111/jcpp. 13104

28. Gadow KD, Sprafkin JN (2015) Child and Adolescent Symptoms Inventory, vol 5. In: Checkmate Plus, Stony Brook New York.

29. Althoff RR, Rettew DC, Ayer LA, Hudziak JJ (2010) Crossinformant agreement of the Dysregulation Profile of the Child Behavior Checklist. Psychiatry Res 178:550-555. https://doi.org/ 10.1016/j.psychres.2010.05.002

30. Weller EB, Weller RA, Fristad MA et al (2000) Children's interview for psychiatric syndromes (ChIPS). J Am Acad Child Adolesc Psychiatry 39:76-84. https://doi.org/10.1097/00004583200001000-00019

31. Sheehan DV, Lecrubier Y, Sheehan KH et al (1998) The MiniInternational Neuropsychiatric Interview (M.I.N.I): The development and validation of a structured diagnostic psychiatric interview for DSM-IV and ICD-10. J Clin Psychiatry 59:22-33

32. Kessler RC, Ustün TB (2004) The World Mental Health (WMH) Survey Initiative Version of the World Health Organization (WHO) Composite International Diagnostic Interview (CIDI). Int J Methods Psychiatr Res 13:93-121. https://doi.org/10.1002/ mpr.168

33. Scheeringa MS, Haslett N (2010) The reliability and criterion validity of the Diagnostic Infant and Preschool Assessment: a new diagnostic instrument for young children. Child Psychiatry Hum Dev 41:299-312. https://doi.org/10.1007/ s10578-009-0169-2

34. Chen Y-L, Shen L-J, Gau SS-F (2017) The Mandarin version of the Kiddie-Schedule for Affective Disorders and SchizophreniaEpidemiological version for DSM-5-a psychometric study. J Formos Med Assoc 116:671-678. https://doi.org/10.1016/j.jfma. 2017.06.013

35. First MB, Spitzer RL, Williams JBW et al (1995) Structured clinical interview for DSM-IV (SCID). Amercian Psychiatric Association, Washington, DC

36. Grau K, Plener PL, Hohmann S et al (2018) Prevalence rate and course of symptoms of disruptive mood dysregulation disorder (DMDD): a population-based study. Z Kinder Jugendpsychiatr Psychother 46:29-38. https://doi.org/10.1024/1422-4917/a0005 52

37. Copeland WE, Simonoff E, Stringaris A (2016) Disruptive mood dysregulation disorder in children with autism spectrum disorder. J Am Acad Child Adolesc Psychiatry 55:S269-S270. https://doi. org/10.1016/j.jaac.2016.07.164

38. de la Peña FR, Rosetti MF, Rodríguez-Delgado A et al (2018) Construct validity and parent-child agreement of the six new or modified disorders included in the Spanish version of the Kiddie Schedule for Affective Disorders and Schizophrenia present and Lifetime Version DSM-5 (K-SADS-PL-5). J Psychiatr Res 101:28-33. https://doi.org/10.1016/j.jpsychires.2018.02.029 
39. Kessel EM, Dougherty LR, Kujawa A et al (2016) Longitudinal associations between preschool disruptive mood dysregulation disorder symptoms and neural reactivity to monetary reward during preadolescence. J Child Adolesc Psychopharmacol 26:131137. https://doi.org/10.1089/cap.2015.0071

40. Ünal F, Öktem F, Çetin Çuhadaroglu F et al (2019) Reliability and validity of the schedule for affective disorders and schizophrenia for school-age children-present and lifetime version, DSM-5 November 2016-Turkish Adaptation (K-SADS-PL-DSM5-T). Turk J Psychiatry. https://doi.org/10.5080/u23408

41. Wiggins JL, Brotman MA, Adleman NE et al (2016) Neural correlates of irritability in disruptive mood dysregulation and bipolar disorders. Am J Psychiatry 173:722-730. https://doi.org/ 10.1176/appi.ajp.2015.15060833

42. Lochman JE, Evans SC, Burke JD et al (2015) An empirically based alternative to DSM-5's disruptive mood dysregulation disorder for ICD-11. World Psychiatry 14:30-33. https://doi.org/10. 1002/wps.20176

43. Roy AK, Lopes V, Klein RG (2014) Disruptive mood dysregulation disorder (DMDD): a new diagnostic approach to chronic irritability in youth. Am J Psychiatry 171:918-924. https://doi. org/10.1176/appi.ajp.2014.13101301

44. Mayes SD, Waxmonsky JD, Calhoun SL, Bixler EO (2016) Disruptive Mood Dysregulation Disorder Symptoms and Association with Oppositional Defiant and Other Disorders in a General Population Child Sample. J Child Adolesc Psychopharmacol 26(2):101-106. https://doi.org/10.1089/cap.2015.0074

45. Laporte PP, Matijasevich A, Munhoz TN et al (2020) Disruptive mood dysregulation disorder: symptomatic and syndromic thresholds and diagnostic operationalization. J Am Acad Child Adolesc Psychiatry. https://doi.org/10.1016/j.jaac.2019.12.008

46. Tseng W-L (2020) Editorial: A transdiagnostic symptom requires a transdiagnostic approach: neural mechanisms of pediatric irritability. J Am Acad Child Adolesc Psychiatry. https://doi.org/10. 1016/j.jaac.2020.09.008

47. Leibenluft E, Stoddard J (2013) The developmental psychopathology of irritability. Dev Psychopathol 25:1473-1487. https:// doi.org/10.1017/S0954579413000722

48. Vidal-Ribas P, Brotman MA, Valdivieso I et al (2016) The status of irritability in psychiatry: a conceptual and quantitative review. J Am Acad Child Adolesc Psychiatry 55:556-570. https://doi. org/10.1016/j.jaac.2016.04.014

49. Haller SP, Stoddard J, MacGillivray C et al (2018) A doubleblind, randomized, placebo-controlled trial of a computer-based Interpretation Bias Training for youth with severe irritability: a study protocol. Trials 19:626. https://doi.org/10.1186/ s13063-018-2960-5

50. Larson R, Csikszentmihalyi M (1992) Validity and reliability of the Experience Sampling Method. In: Csikszentmihalyi M, Vries M (eds) The Experience of Psychopathology: Investigating Mental Disorders in their Natural Settings (pp. 43- 57). Cambridge: Cambridge University Press. https://doi.org/10.1017/CBO97 80511663246.006

51. Meyers E, DeSerisy M, Roy AK (2017) Disruptive mood dysregulation disorder (DMDD): an RDoC perspective. J Affect Disord 216:117-122. https://doi.org/10.1016/j.jad.2016.08.007

52. Matarazzo JD (1990) Psychological assessment versus psychological testing. Validation from Binet to the school, clinic, and courtroom. Am Psychol 45:999-1017. https://doi.org/10.1037// 0003-066x.45.9.999

53. McTate EA, Leffler JM (2017) Diagnosing disruptive mood dysregulation disorder: integrating semi-structured and unstructured interviews. Clin Child Psychol Psychiatry 22:187-203. https:// doi.org/10.1177/1359104516658190

54. Dougherty LR, Smith VC, Bufferd SJ et al (2014) DSM-5 disruptive mood dysregulation disorder: correlates and predictors in young children. Psychol Med 44:2339-2350. https://doi.org/ 10.1017/S0033291713003115

55. Parmar A, Vats D, Parmar R, Aligeti M (2014) Role of naltrexone in management of behavioral outbursts in an adolescent male diagnosed with disruptive mood dysregulation disorder. J Child Adolesc Psychopharmacol 24:594-595. https://doi.org/10.1089/ cap.2014.0072

56. Sparks GM, Axelson DA, Yu H et al (2014) Disruptive mood dysregulation disorder and chronic irritability in youth at familial risk for bipolar disorder. J Am Acad Child Adolesc Psychiatry 53:408-416. https://doi.org/10.1016/j.jaac.2013.12.026

57. Deveney CM, Hommer RE, Reeves E et al (2015) A prospective study of severe irritability in youths: 2- and 4-year follow-up. Depress Anxiety 32:364-372. https://doi.org/10.1002/da.22336

58. Estrada Prat X, Alvarez Guerrico I, Camprodon Rosanas E et al (2015) Disruptive mood dysregulation disorder and pediatric bipolar disorder. Sleep and attention. Eur Child Adolesc Psych 24:S245-S245

59. Mitchell RHB, Hlastala SA, Mufson L, et al (2015) Correlates of disruptive mood dysregulated disorder (DMDD) phenotype among adolescents with bipolar disorder. 17th Annual Conference of the International Society for Bipolar Disorders, June 3 -6, Toronto, Canada. Bipolar Disorders, Volume 17, S1. https:// doi.org/10.1111/bdi.12309

60. Schilpzand EJ, Hazell P, Nicholson J, et al (2015) Comorbidity and correlates of disruptive mood dysregulation disorder in 6-8 year old children with ADHD. 5th World Congress on ADHD: From Child to Adult Disorder: 28th-31st May, Glasgow Scotland. Atten Defic Hyperact Disord. Volume 7, Suppl 1:1-119. https:// doi.org/10.1007/s12402-015-0169-y

61. Stoddard J, Sharif-Askary B, Harkins E et al (2015) Preliminary evidence for computer-based training targeting hostile interpretation bias as a treatment for DMDD. Neuropsychopharmacology 40:S290-S291

62. Tseng W-L, Brotman M, Deveney C et al (2015) Neural mechanisms of irritability in youth across diagnoses: dimensional and categorical approaches. Neuropsychopharmacology. Nature Publishing Group, London, pp S190-S191

63. Uran P, Kılıç BG (2015) Family Functioning, Comorbidities, and behavioral profiles of Children with ADHD and disruptive mood dysregulation disorder. J Atten Disord. https://doi.org/10.1177/ 1087054715588949

64. Uran P, Kilic B (2015) Comparison of family functioning and psychiatric comorbidities of children with attention deficit hyperactivity disorder and disruptive mood dysregulation disorder. Eur Child Adolesc Psychiatry 24:S140-S140

65. Averna R, D'Agati E, Vicari S (2016) Low-dose aripiprazole monotherapy in a young child with disruptive mood dysregulation disorder. Ther Adv 6:149-151. https://doi.org/10.1177/ 2045125315591927

66. Baweja R, Belin PJ, Humphrey HH et al (2016) The effectiveness and tolerability of central nervous system stimulants in schoolage children with attention-deficit/hyperactivity disorder and disruptive mood dysregulation disorder across home and school. J Child Adolesc Psychopharmacol 26:154-163. https://doi.org/10. 1089/cap.2015.0053

67. Brotman MA, Tseng W-L, Wiggins J et al (2016) Neural correlates of attention bias in irritability and anxiety. The American College of Neuropsychopharmacology (ACNP) 56th Annual Meeting Poster Session, December 3-7 2017, Volume 42. https:// doi.org/10.1038/npp.2017.264

68. Carlson GA, Barrios CS, Dougherty LR, Klein DN (2016) Stability and predictors of disruptive mood dysregulation disorder in young children. J Am Acad Child Adolesc Psychiatry 55:S270. https://doi.org/10.1016/j.jaac.2016.07.166 
69. Dougherty LR, Smith VC, Bufferd SJ et al (2016) Disruptive mood dysregulation disorder at the age of 6 years and clinical and functional outcomes 3 years later. Psychol Med 46:1103-1114. https://doi.org/10.1017/S0033291715002809

70. Freeman AJ, Youngstrom EA, Youngstrom JK, Findling RL (2016) Disruptive mood dysregulation disorder in a community mental health clinic: prevalence, comorbidity and correlates. $\mathbf{J}$ Child Adolesc Psychopharmacol 26:123-130. https://doi.org/10. 1089/cap.2015.0061

71. Fristad MA, Wolfson H, Algorta GP et al (2016) Disruptive mood dysregulation disorder and bipolar disorder not otherwise specified: fraternal or identical twins? J Child Adolesc Psychopharmacol 26:138-146. https://doi.org/10.1089/cap.2015.0062

72. Gold AL, Brotman MA, Adleman NE et al (2016) Comparing brain morphometry across multiple childhood psychiatric disorders. J Am Acad Child Adolesc Psychiatry 55:1027-1037.e3. https://doi.org/10.1016/j.jaac.2016.08.008

73. Kilic O, Demirbas Cakir E, Tufan AE (2016) Disruptive mood dysregulation disorder in adults: a case report. Eur psychiatr 33:S363-S363. https://doi.org/10.1016/j.eurpsy.2016.01.1302

74. Mitchell RHB, Timmins V, Collins J et al (2016) Prevalence and correlates of disruptive mood dysregulation disorder among adolescents with bipolar disorder. J Child Adolesc Psychopharmacol 26:147-153. https://doi.org/10.1089/cap.2015.0063

75. Mulraney M, Schilpzand EJ, Hazell P et al (2016) Comorbidity and correlates of disruptive mood dysregulation disorder in 6-8-year-old children with ADHD. Eur Child Adolesc Psychiatry 25:321-330. https://doi.org/10.1007/s00787-015-0738-9

76. Pogge DL, Chase D, Buccolo M et al (2016) Prevalence of and comorbidities with disruptive mood dysregulation disorder in an inpatient setting. The Scientific Proceedings of the 63th Annual Meeting of the American Academy of Child \& Adolescent Psychiatry Journal of the American Academy of Child \& Adolescent Psychiatry, Volume 55, Issue 10S

77. Stoddard J, Sharif-Askary B, Harkins EA et al (2016) An open pilot study of training hostile interpretation bias to treat disruptive mood dysregulation disorder. J Child Adolesc Psychopharmacol 26:49-57. https://doi.org/10.1089/cap.2015.0100

78. Stoddard J (2016) Transdiagnostic neural mechanisms of irritability. J Am Acad Child Adolesc Psychiatry 55:S332. https://doi. org/10.1016/j.jaac.2016.07.393

79. Taskiran S, Mutluer T, Sanli I et al (2016) The correlation between distractorincorporated continuous performance test and neuropsychological test battery results of children with attentiondeficit/hyperactivity disorder. J Am Acad Child Adolesc Psychiatry 55:S214. https://doi.org/10.1016/j.jaac.2016.09.351

80. Tiwari R, Agarwal V, Arya A et al (2016) An exploratory clinical study of disruptive mood dysregulation disorder in children and adolescents from India. Asian J Psychiatr 21:37-40. https://doi. org/10.1016/j.ajp.2016.02.005

81. Topal Z, Demir N, Tuman TC et al (2016) Rates of disruptive mood dysregulation disorder among adolescent offspring of parents with recurrent major depressive disorder versus those with bipolar disorder and matched healthy controls. J Am Acad Child Adolesc Psychiatry 55:S190. https://doi.org/10.1016/j.jaac.2016. 09.280

82. Topal Z, Demir N, Tuman TC et al (2016) Rates of disruptive mood dysregulation disorder in adolescent children of parents with recurrent depression or bipolar disorder and healthy controls. Klinik Psikofarmakoloji Bulteni 26:S11-S12

83. Tudor ME, Ibrahim K, Bertschinger E et al (2016) Cognitivebehavioral therapy for a 9-year-old girl with disruptive mood dysregulation disorder. Clin Case Stud 15:459-475. https://doi. org/10.1177/1534650116669431

84. Tufan E, Topal Z, Demir N et al (2016) Sociodemographic and clinical features of disruptive mood dysregulation disorder: a chart review. J Child Adolesc Psychopharmacol 26:94-100. https://doi.org/10.1089/cap.2015.0004

85. Alexander LM, Salum GA, Swanson JM, Milham MP (2017) Development of the Extended Strengths and Weaknesses Assessment of Normal Behavior Rating Scale (E-SWAN). The Scientific Proceedings of the 64th Annual Meeting of the American Academy of Child \& Adolescent Psychiatry Journal of the American Academy of Child \& Adolescent Psychiatry, Volume 56 , Issue $10 \mathrm{~S}$

86. Dougherty LR, Barrios CS, Carlson GA, Klein DN (2017) Predictors of later psychopathology in young children with disruptive mood dysregulation disorder. J Child Adolesc Psychopharmacol 27:396-402. https://doi.org/10.1089/cap.2016.0144

87. Estrada Prat X, Álvarez-Guerrico I, Bleda-Hernández MJ et al (2017) Sleep study in disruptive mood dysregulation disorder and bipolar children. Actas Esp Psiquiatr 45:12-20

88. Eyre O, Langley K, Stringaris A et al (2017) Irritability in ADHD: associations with depression liability. J Affect Disord 215:281-287. https://doi.org/10.1016/j.jad.2017.03.050

89. Faheem S, Petti V, Mellos G (2017) Disruptive mood dysregulation disorder and its effect on bipolar disorder. Ann Clin Psychiatry 29:84-91

90. Higdon C, Fornari V, Sheridan E, et al (2017) Conducting a multi-site, community-based, pragmatic research trial: study design, recruitment barriers, and initial sample characteristics of mobility. The Scientific Proceedings of the 64th Annual Meeting of the American Academy of Child \& Adolescent Psychiatry Journal of the American Academy of Child \& Adolescent Psychiatry, Volume 56, Issue $10 \mathrm{~S}$

91. Jain U (2017) The use of guanfacine (Intuniv XR) in the treatment of disruptive mood dysregulation disorder-Clinical experience from telepsychiatry. 25th European Congress of Psychiatry, European Psychiatry, Volume 41S, S1-S910. https://doi.org/ 10.1016/j.eurpsy.2017.01.449

92. Jalnapurkar IR, Desai P, Pemberton AM et al (2017) Stressors and aggressors: violent aggression often precedes inpatient admission and exacerbates caregiver stress. J Am Acad Child Adolesc Psychiatry 56:S171. https://doi.org/10.1016/j.jaac.2017. 09.069

93. Kircanski K, White L, Tseng W-L, et al (2017) Computational phenotyping reveals a double dissociation in the neural mechanisms of irritability and anxiety in youth. The American College of Neuropsychopharmacology (ACNP) 56th Annual Meeting Poster Session, December 3-7 2017, Volume 42. https:// doi.org/10.1038/npp.2017.264

94. Kircanski K, White L, Tseng W-L, et al (2017) Shared and unique neural correlates of threat processing in pediatric irritability and anxiety. 72nd Annual Scientific Convention and Meeting of Biological Psychiatry, Journal of Biological Psychiatry Volume 81, Issue 10S

95. Le JF, Lohr WD, Feygin YB et al (2017) Examining trends and interactions in the diagnoses of pediatric bipolar disorders and disruptive mood dysregulation disorder (DMDD) in Kentucky Children and Adolescents Receiving Medicaid. The Scientific Proceedings of the 64th Annual Meeting of the American Academy of Child \& Adolescent Psychiatry Journal of the American Academy of Child \& Adolescent Psychiatry, Volume 56, Issue 10S

96. Martin SE, Hunt JI, Mernick LR et al (2017) Temper loss and persistent irritability in preschoolers: implications for diagnosing disruptive mood dysregulation disorder in early childhood. Child Psychiatry Hum Dev 48:498-508. https://doi.org/10.1007/ s10578-016-0676-x

97. Matthews DT, Matthews GW (2017) Disruptive mood dysregulation disorder: Medical management without the use of antipsychotics. 2016 NEI Psychopharmacology Congress. CNS 
Spectrums 22(1):62-109. https://doi.org/10.1017/S109285291 6000900

98. Mitchell PB, Perich T, Frankland A et al (2017) Irritability and disruptive mood dysregulation disorder as potential precursors of bipolar disorder. Bipolar Disorders. Wiley, Hoboken, pp 48-49

99. Munhoz TN, Santos IS, Barros AJD et al (2017) Perinatal and postnatal risk factors for disruptive mood dysregulation disorder at age 11: 2004 Pelotas Birth Cohort Study. J Affect Disord 215:263-268. https://doi.org/10.1016/j.jad.2017.03.040

100. Özyurt G, Emiroglu N, Baykara B, Akay Pekcanlar A (2017) Effectiveness and adverse effects of methylphenidate treatment in children diagnosed with disruptive mood dysregulation disorder and attention-deficit hyperactivity disorder: a preliminary report. Psychiatry Clin Psychopharmacol 27:99-100. https://doi.org/10. 1080/24750573.2017.1293252

101. Pagliaccio D, Wiggins JL, Adleman NE et al (2017) Behavioral and neural sustained attention deficits in disruptive mood dysregulation disorder and attention-deficit/hyperactivity disorder. J Am Acad Child Adolesc Psychiatry 56:426-435. https://doi.org/ 10.1016/j.jaac.2017.02.008

102. Perepletchikova F, Nathanson D, Axelrod SR et al (2017) Randomized clinical trial of dialectical behavior therapy for preadolescent children with disruptive mood dysregulation disorder: feasibility and outcomes. J Am Acad Child Adolesc Psychiatry 56:832-840. https://doi.org/10.1016/j.jaac.2017.07.789

103. Perhamus G, Kircanski K, Lee Wiggins J et al (2017) Face emotion labeling in pediatric irritability: behavioral and neural correlates. The Scientific Proceedings of the 64th Annual Meeting of the American Academy of Child \& Adolescent Psychiatry Journal of the American Academy of Child \& Adolescent Psychiatry, Volume 56, Issue $10 \mathrm{~S}$

104. Propper L, Cumby J, Patterson VC et al (2017) Disruptive mood dysregulation disorder in offspring of parents with depression and bipolar disorder. Br J Psychiatry 210:408-412. https://doi. org/10.1192/bjp.bp.117.198754

105. Ramires VRR, Godinho LBR, Goodman G (2017) The therapeutic process of a child diagnosed with disruptive mood dysregulation disorder. Psychoanal Psychol 34:488-498. https://doi.org/10. 1037/pap0000134

106. Stoddard J, Tseng W-L, Kim P et al (2017) Association of irritability and anxiety with the neural mechanisms of implicit face emotion processing in youths with psychopathology. JAMA Psychiat 74:95. https://doi.org/10.1001/jamapsychiatry.2016.3282

107. Stoddard J, Jones M, Haller S et al (2017) Identifying the mechanisms of interpretation bias in irritability. J Am Acad Child Adolesc Psychiatry 56:S324-S325. https://doi.org/10.1016/j. jaac.2017.07.664

108. Swetlitz C, Averbeck B, Leibenluft E et al (2017) Exploreexploit decision making: differences in information-seeking behavior in pediatric psychopathology. J Am Acad Child Adolesc Psychiatry 56:S233. https://doi.org/10.1016/j.jaac.2017. 09.231

109. Taskiran S, Mutluer T, Necef I (2017) Neuropsychological profile differences between children with disruptive mood dysregulation disorder (DMDD) and attention-deficit/hyperactivity disorder (ADHD): a preliminary study. J Am Acad Child Adolesc Psychiatry 56:S233-S234. https://doi.org/10. 1016/j.jaac.2017.09.232

110. Taskiran S, Turkakin E, Karamanci C et al (2017) Face emotion recognition differences with respect to frustration in disruptive mood dysregulation disorder (DMDD) and attention-deficit/ hyperactivity disorder (ADHD). J Am Acad Child Adolesc Psychiatry 56:S234. https://doi.org/10.1016/j.jaac.2017.09.233

111. Tseng W-L, Deveney C, Brotman M et al (2017) Neural mechanisms of frustration and irritability across diagnoses. 72nd Annual Scientific Convention and Meeting of Biological
Psychiatry, Journal of Biological Psychiatry Volume 81, Issue $10 \mathrm{~S}$

112. Waxmonsky JG, Waschbusch D, Babocsai L, Belin P (2017) Assessment and treatment of hostile attribution bias in children with disruptive mood dysregulation disorder. J Am Acad Child Adolesc Psychiatry 56:S246. https://doi.org/10.1016/j. jaac.2017.09.267

113. Abouzed M, Elawady A (2018) Disruptive mood dysregulation disorder in offspring of parents with ADHD. E-Poster Walk. European Psychiatry, 48(S1):S141-S358. https://doi.org/10. 1016/j.eurpsy.2017.12.016

114. Bryant B, Bear MH, Rowlett JK (2018) DMDD patients with and without a history of childhood abuse and/or neglect: comparison of hospital length of stay, use of antipsychotics, and restraints. The Scientific Proceedings of the 65th Annual Meeting of the American Academy of Child \& Adolescent Psychiatry, Journal of the American Academy of Child \& Adolescent Psychiatry, Volume 57, Issue 10S

115. Cuffe SP, Glassman RS, Singh K, Holbrook J (2018) Identification of bipolar DMDD in a school-based study of school-aged children and adolescents. The Scientific Proceedings of the 65th Annual Meeting of the American Academy of Child \& Adolescent Psychiatry, Journal of the American Academy of Child \& Adolescent Psychiatry, Volume 57, Issue 10S

116. Delaplace R, Garny de La Rivière S, Bon Saint Come M et al (2018) Sleep and disruptive mood dysregulation disorder: a pilot actigraphy study. Arch Pediatr. https://doi.org/10.1016/j.arcped. 2018.05.003

117. Fridson R, Bailey S, Edwards E, et al (2018) Comparison of prescribed pharmacotherapy of patients diagnosed with DMDD versus bipolar disorder in child and adolescent psychiatric outpatients. The Scientific Proceedings of the 65th Annual Meeting of the American Academy of Child \& Adolescent Psychiatry, Journal of the American Academy of Child \& Adolescent Psychiatry, Volume 57, Issue $10 \mathrm{~S}$

118. Kircanski K, White LK, Tseng W-L et al (2018) A latent variable approach to differentiating neural mechanisms of irritability and anxiety in youth. JAMA Psychiat 75:631. https://doi.org/10. 1001/jamapsychiatry.2018.0468

119. Miller L, Hlastala SA, Mufson L et al (2018) Interpersonal psychotherapy for mood and behavior dysregulation: pilot randomized trial. Depress Anxiety 35:574-582. https://doi.org/10. 1002/da.22761

120. Mroczkowski MM, McReynolds LS, Fisher P, Wasserman GA (2018) Disruptive mood dysregulation disorder in juvenile justice. J Am Acad Psychiatry Law 46:329-338. https://doi.org/10. 29158/JAAPL.003767-18

121. Pan P-Y, Fu A-T, Yeh C-B (2018) Aripiprazole/methylphenidate combination in children and adolescents with disruptive mood dysregulation disorder and attention-deficit/hyperactivity disorder: an open-label study. J Child Adolesc Psychopharmacol 28:682-689. https://doi.org/10.1089/cap.2018.0068

122. Sagar-Ouriaghli I, Milavic G, Barton R et al (2018) Comparing the DSM-5 construct of Disruptive Mood Dysregulation Disorder and ICD-10 Mixed disorder of emotion and conduct in the UK Longitudinal Assessment of manic symptoms (UK-LAMS) Study. Eur Child Adolesc Psychiatry 27:1095-1104. https://doi. org/10.1007/s00787-018-1149-5

123. Vidal-Ribas P, Brotman MA, Salum GA et al (2018) Deficits in emotion recognition are associated with depressive symptoms in youth with disruptive mood dysregulation disorder. Depress Anxiety 35:1207-1217. https://doi.org/10.1002/da.22810

124. Walyzada F, Manocha P, Odom C et al (2018) Prescribing practices of antipsychotics in children and adolescents with a diagnosis of DMDD in an outpatient setting. Journal of the American 
Academy of Child \& Adolescent Psychiatry, 579(10):Supplement p. S170. https://doi.org/10.1016/j.jaac.2018.09.123.

125. Wiggins JL, Briggs-Gowan MJ, Estabrook R et al (2018) Identifying clinically significant irritability in early childhood. J Am Acad Child Adolesc Psychiatry 57:191-199.e2. https://doi.org/ 10.1016/j.jaac.2017.12.008

126. Winters DE, Fukui S, Leibenluft E, Hulvershorn LA (2018) Improvements in irritability with open-label methylphenidate treatment in youth with comorbid attention deficit/hyperactivity disorder and disruptive mood dysregulation disorder. J Child Adolesc Psychopharmacol 28:298-305. https://doi.org/10.1089/ cap.2017.0124

127. Basu S, Isaacs AN (2019) Profile of transcultural patients in a regional Child and Adolescent Mental Health Service in Gippsland, Australia: the need for a multidimensional understanding of the complexities. Int J Soc Psychiatry 65:217-224. https://doi. org/10.1177/0020764019835264

128. Benarous X, Ferrafiat V, Zammit J et al (2019) Effective use of atomoxetine to treat six inpatient youths with disruptive mood dysregulation disorder without attention deficit disorder. CNS Spectr. https://doi.org/10.1017/S1092852919001020

129. Benarous X (2019) Are youths with DMDD different from youths with other depressive disorders? A retrospective chart review. The Scientific Proceedings of the 66th Annual Meeting of the American Academy of Child \& Adolescent Psychiatry Journal of the American Academy of Child \& Adolescent Psychiatry, Volume 58, Issue 10

130. Chen Y-L, Chen WJ, Lin K-C et al (2019) Prevalence of DSM-5 mental disorders in a nationally representative sample of children in Taiwan: methodology and main findings. Epidemiol Psychiatr Sci 29:e15. https://doi.org/10.1017/S2045796018000793

131. Eyre O, Riglin L, Leibenluft E et al (2019) Irritability in ADHD: association with later depression symptoms. Eur Child Adolesc Psychiatry 28:1375-1384. https://doi.org/10.1007/ s00787-019-01303-x

132. Guilé J-M (2019) Sleep abnormalities in disruptive mood dysregulation disorder: an actigraphy study. J Am Acad Child Adolesc Psychiatry 58:S353. https://doi.org/10.1016/j.jaac.2019.07.851

133. Haller SP, Jones M, Pine D, Leibenluft E, Brotman MA, Stoddard J (2019) A computational model to measure mechanisms of interpretation bias training for treating disruptive mood dysregulation disorder. Biological Psychiatry 85(10)Suppl:S69. https://doi.org/ 10.1016/j.biopsych.2019.03.180

134. Ignaszewski MJ, Munshi K, Fogler J, Augustyn M (2019) Transitions, suicidality, and underappreciated autism spectrum disorder in a high school student. J Dev Behav Pediatrics 40:563-565. https://doi.org/10.1097/DBP.0000000000000717

135. Linke JO, Kircanski K, Brooks J et al (2019) Exposure-based cognitive-behavioral therapy for disruptive mood dysregulation disorder: an evidence-based case study. Behav Ther 51:320-333. https://doi.org/10.1016/j.beth.2019.05.007

136. Linke JO, Adleman NE, Sarlls J et al (2019) White matter microstructure in pediatric bipolar disorder and disruptive mood dysregulation disorder. J Am Acad Child Adolesc Psychiatry. https:// doi.org/10.1016/j.jaac.2019.05.035

137. Mulraney M, Silk TJ, Efron D et al (2019) Persistence and neural correlates of disruptive mood dysregulation disorder in 10-yearold children with ADHD. 7th World Congress on ADHD: From
Child to Adult Disorder: 25th-28th April, Lisbon Portugal. Atten Defic Hyperact Disord; Volume 11, Suppl 1:1-89. https://doi.org/ 10.1007/s12402-019-00295-7

138. Rice T, Simon H, Barcak D et al (2019) Amantadine for treatment of disruptive mood dysregulation disorder symptoms. J Child Adolesc Psychopharmacol 29:642-646. https://doi.org/ 10.1089/cap.2019.29172.bjc

139. Towbin K, Vidal-Ribas P, Brotman MA et al (2019) A doubleblind randomized placebo-controlled trial of citalopram adjunctive to stimulant medication in youth with chronic severe irritability. J Am Acad Child Adolesc Psychiatry 59:350-361. https:// doi.org/10.1016/j.jaac.2019.05.015

140. Tseng W-L, Deveney CM, Stoddard J et al (2019) Brain mechanisms of attention orienting following frustration: associations with irritability and age in youths. Am J Psychiatry 176:67-76. https://doi.org/10.1176/appi.ajp.2018.18040491

141. Tüğen LE, Göksu M, Burcu Ayaz A (2020) Disruptive mood dysregulation disorder in a primary school sample. Asian J Psychiatr 48:101858. https://doi.org/10.1016/j.ajp.2019.101858

142. Benarous X, Bury V, Lahaye $\mathrm{H}$ et al (2020) Sensory processing difficulties in youths with disruptive mood dysregulation disorder. Front Psychiatry 11:164. https://doi.org/10.3389/fpsyt.2020. 00164

143. Benarous X, Renaud J, Breton JJ et al (2020) Are youths with disruptive mood dysregulation disorder different from youths with major depressive disorder or persistent depressive disorder? J Affect Disord 265:207-215. https://doi.org/10.1016/j.jad.2020. 01.020

144. Chang C-H, Chang Y-C, Cheng H, Tzang R-F (2020) Treatment efficacy of internet gaming disorder with attention deficit hyperactivity disorder and emotional dysregulaton. Int J Neuropsychopharmacol. https://doi.org/10.1093/ijnp/pyaa010

145. Cimino S, Carola V, Cerniglia L et al (2020) The $\mu$-opioid receptor gene $\mathrm{A} 118 \mathrm{G}$ polymorphism is associated with insecure attachment in children with disruptive mood regulation disorder and their mothers. Brain Behav. https://doi.org/10.1002/brb3. 1659

146. Haller SP, Kircanski K, Stringaris A et al (2020) The clinician affective reactivity index: validity and reliability of a clinicianrated assessment of irritability. Behav Ther 51:283-293. https:// doi.org/10.1016/j.beth.2019.10.005

147. Haller SP, Stoddard J, Pagliaccio D et al (2020) Computational modeling of attentional impairments in disruptive mood dysregulation and attention deficit/hyperactivity disorder. Biol Psychiat 87:S155-S156. https://doi.org/10.1016/j.biopsych.2020.02.412

148. Johnstone JM, Leung BMY, Srikanth P et al (2020) Development of a composite primary outcome score for children with attention-deficit/hyperactivity disorder and emotional dysregulation. J Child Adolesc Psychopharmacol 30:166-172. https://doi. org/10.1089/cap.2019.0179

149. Le JF, Feygin Y, Creel L et al (2020) Trends in diagnosis of bipolar and disruptive mood dysregulation disorders in children and youth. J Affect Disord 264:242-248. https://doi.org/10.1016/j. jad.2019.12.018

150. Tseng W-L, Scheinost D, Pine DS et al (2020) Functional connectivity during frustration is predictive of irritability in youth. Biol Psychiat 87:S108. https://doi.org/10.1016/j.biopsych.2020. 02.297 